\title{
Measurement-based support for post-earthquake assessment of buildings
}

\author{
Yves Reuland ${ }^{\mathrm{a}}$, Pierino Lestuzzi ${ }^{\mathrm{a}}$ and Ian F. C. Smith ${ }^{\mathrm{a}}$ \\ a Applied Computing and Mechanics Laboratory (IMAC), School of Architecture, Civil and \\ Environmental Engineering (ENAC), Swiss Federal Institute of Technology (EPFL), Lausanne, \\ Switzerland
}

\author{
ARTICLE HISTORY \\ Compiled September 14, 2018
}

\begin{abstract}
After a damaging earthquake, assessment of the residual seismic capacity is required for large parts of the building stock. Increased vulnerability of structures together with the threat of immediate aftershocks call for rapid and objective decision making. Structural identification has the potential to reduce parameter-value uncertainties of physics-based models through interpreting measurement data. Significant amounts of uncertainty are associated with the non-linear behaviour of structures during extreme events such as earthquakes. Therefore, a structural identification methodology that accommodates multiple sources of systematic modelling uncertainties is used. Error-domain model falsification (EDMF) enables structural identification through combining damage grades observed by visual inspection with fundamental frequencies that are derived from ambient vibrations. Parametric uncertainties of a hysteretic model are reduced with the two information sources in order to extrapolate the vulnerability of the building regarding future earthquakes. The applicability of the methodology is shown using measurements made on a mixed reinforced-concrete unreinforced-masonry building tested on a shaking table. Based on nonlinear timehistory analyses involving single-degree-of-freedom models, EDMF leads to more precise, yet robust, vulnerability predictions of earthquake-damaged buildings when compared with prediction ranges that are obtained without data interpretation.
\end{abstract}

\section{KEYWORDS}

Post-earthquake assessment; residual seismic capacity; model-based data interpretation; structural identification; visual inspection; ambient-vibration measurements

\section{Introduction}

The assessment of deteriorated structures after a damaging earthquake is a challenging task that is further complicated by the need for rapid decision making resulting from the threat of aftershocks. Current practice involves visual inspections that are performed by certified experts. The goal of such inspections is to tag buildings as either safe or unsafe for occupancy (Yeo \& Cornell, 2009). Due to the relative slowness of building inspections, important societal and financial losses may result from the unavailability of structures to either harbour the affected population or to generate economic activity. Also, despite being thoroughly standardized (through codes such as ATC20 in America 
and AeDES in Italy (Baggio et al., 2007)), visual inspection outcomes are prone to much subjectivity (Galloway et al., 2014; Goretti \& Di Pasquale, 2002; Goretti, Hutt, \& Hedelund, 2017). In addition, slightly damaged buildings may have significantly increased vulnerability during aftershocks (Esteva, Díaz-López, Vásquez, \& León, 2016; Ghosh, Padgett, \& Sánchez-Silva, 2015; Jalayer, Asprone, Prota, \& Manfredi, 2011; Jalayer \& Ebrahimian, 2017; Raghunandan, Liel, \& Luco, 2015). Such increased vulnerability is not easily captured by visual inspections that mainly provide estimations of structural performance during the main shock (Marshall et al., 2013). In addition, difficulties in establishing residual capacity of earthquake-damaged buildings have contributed to the decision of demolishing buildings (Elwood et al., 2016), which is ecologically unsustainable. Recently, use of unmanned aerial vehicles to perform rapid post-earthquake surveys has been proposed (Vona, Cascini, Mastroberti, Murgante, \& Nolè, 2017) to enhance visual inspection. However, determining residual capacities of deteriorated buildings remains challenging (Marquis, Kim, Elwood, \& Chang, 2017).

By combining physics-based models and measurements, structural identification techniques can help to overcome the shortcomings of relying mainly on visual inspection. Structural identification in a post-seismic context is defined as the task of improving knowledge related to the structural state (by the means of a physical model) through interpreting measurement data. Physics-based models allow the engineer to predict the behaviour of a structure under actions or conditions that differ from the measurement context. Such extrapolations are essential for predicting the structural response during future earthquakes from measurement data that is gathered under different conditions. Thus, the objective of structural identification is to reduce parametric uncertainty of physics-based models. In addition, relying on values taken from codes has been shown to produce over-conservative results (D'Ayala \& Paganoni, 2010).

The use of measurement data to assess earthquake-damaged buildings has been proposed in the past (Dunand et al., 2004). The aim of taking measurements is to reduce the time that is needed for assessment and to provide quantifiable support for decision-making. Ambient-vibration measurements provide non-destructive testing data that can be obtained quickly at a reasonable expense. Although subject to much debate (Behmanesh \& Moaveni, 2015; Gentile \& Messina, 2003; Moaveni, Conte, \& Hemez, 2009), modal parameters derived from ambient vibrations, and more particularly fundamental frequencies, have been shown to be indicators of structural damage (Astorga, Guéguen, \& Kashima, 2018; Calvi, Pinho, \& Crowley, 2006; Clinton, Bradford, Heaton, \& Favela, 2006; Katsanos, Sextos, \& Elnashai, 2014; Michel, Zapico, Lestuzzi, Molina, \& Weber, 2011; Mucciarelli et al., 2004; Vidal, Navarro, Aranda, \& Enomoto, 2013).

In the past, many applications of model updating for damage detection and quantification based on ambient vibration measurements have been proposed (Chellini, De Roeck, Nardini, \& Salvatore, 2010; Moaveni, He, Conte, \& Restrepo, 2010; Shiradhonkar \& Shrikhande, 2011). Also, non-parametric models, such as neural networks(Huang, Hung, Wen, \& Tu, 2003; Maity \& Saha, 2004; Zapico \& Gonzalez, 2006) or continuous wavelet transform (Balafas \& Kiremidjian, 2015), were used to determine the occurrence of structural damage from earthquakes . Goulet, Michel, and Kiureghian (2015) proposed a city-scale non-parametric rapid assessment methodology based on natural frequencies derived from ambient vibrations. Bensi, Kiureghian, and Straub (2014) have integrated Bayesian network and influence diagram into a framework that allows decision-making for transportation networks. This framework involves probabilistic updating using incomplete information from sensors (on structures and in a seismic network) and inspection. Özer and Soyöz (2013) assessed the residual capacity of 
earthquake-damaged reinforced-concrete bridge columns through updated fragility curves and pointed out the importance of structural identification for residual-capacity estimation. Trevlopoulos and Guéguen (2016) proposed an operational forecasting of vulnerability through a sequence of aftershocks based on measured period elongation. Beside these contributions, few research applications on structural identification of damaged buildings to predict future behaviour have been reported.

Increased vulnerability of main-shock damaged buildings with respect to aftershocks was assessed by Iervolino, Giorgio, and Chioccarelli (2014). Jeon, DesRoches, Lowes, and Brilakis (2015) proposed aftershock vulnerability curves. In addition, Raghunandan et al. (2015) estimated the residual capacity of a frame structure after undergoing a main shock without using any data-interpretation technique. Polese, Di Ludovico, Marcolini, Prota, and Manfredi (2015) proposed a simplified tool to estimate the performance loss of buildings due to earthquakes, considering lateral-load-resisting parameters as random variable that is not updated using measurements. Few measurement-based structural identification studies were performed to reduce the uncertainty of building states. In addition, most applications treated reinforced concrete or steel moment frames; very few applications have focused on mixed reinforced-concrete unreinforced-masonry structures. Yazgan and Dazio (2012) assessed the peak displacement experienced by a damaged structure during the earthquakes based on a Bayesian updating scheme for visual inspection (subjective data source) and residual displacement measurements (objective data source). Aside from this work, few proposals combine subjective and objective data sources in structural identification of earthquake-damaged buildings. Jalayer et al. (2011) suggested to use a Bayesian updating framework to update the short-term increase in seismic hazard following a main shock using data from seismic network to calculate structural reliability of main-shock damaged buildings without updating parameters of the structure. Mitrani-Resier, Wu, and Beck (2016) introduced a "virtual inspector" to help experts in tagging buildings after an earthquake based on precise knowledge of the earthquake and detailed modelling of the structure. Use of discrete measurements after an earthquake to update post-seismic residual capacity was not investigated.

In this paper, a methodology for assessing earthquake-damaged buildings is presented. The methodology, based on the error-domain model-falsification (EDMF) framework, allows engineers to combine data from visual inspection with ambient-vibration measurements in order to improve the knowledge of earthquake-damaged structures quickly and at low cost. Identified model instances are used to predict the vulnerability of the building with regard to aftershocks or future earthquake actions. In a case study, the proposed methodology is applied to a mixed reinforced-concrete unreinforced-masonry structure. The structure has been tested on a shaking table and the predicted behaviour is compared to the building response observed during multiple shake-table tests.

The paper starts with a description of a measurement-based structural-identification methodology for earthquake-damaged buildings (diagnosis task, Section 2.2) based on EDMF (Section 2.1). In the following, a methodology to extrapolate structural predictions for vulnerability prognosis tasks is introduced (Section 2.3). The methodology is then applied to a mixed reinforced-concrete unreinforced-masonry specimen tested on a shake table (Section 3). Finally, limitations of the reported case study are discussed (Section 4) and conclusions are presented (Section 5). 


\section{Model-based data interpretation of earthquake-damaged buildings}

\subsection{Data interpretation based on error-domain model falsification}

In the context of the presented post-earthquake-assessment methodology, the aim of implementing model-based data interpretation is to provide estimates of parameter values, which define the structural behaviour under seismic actions. Thereby, the uncertainty related to the residual capacity of earthquake-damaged structures can be reduced. The framework relies on linear elastic measurements (ambient vibrations) and damage grades observed by visual inspection to predict seismic behaviour. Such predictions involve model extrapolation from observed linear behaviour to predicted nonlinear behaviour.

In the past, data-interpretation techniques using residual minimization have been extensively used to estimate optimal parameter values. Such optimal parameter values are then used to perform predictions. More recently, probabilistic approaches for model-based data interpretation that rely on Bayesian conditional probabilites have led to parameter-value estimates that take the form of probability density functions (Beck \& Yuen, 2004; Enright \& Frangopol, 1999). Often, these density functions point towards a maximum likelihood estimate for each parameter. Large and changing model uncertainties that are related to post-earthquake assessment and nonlinear predictions as well as the unknown and changing correlation between error values of coupled predictions undermine the robustness of data-interpretation techniques. This is especially true for techniques that build upon the hypothesis of uncorrelated zero-mean uncertainty distributions for all measured quantities (Reuland, Lestuzzi, \& Smith, 2017).

In order to achieve robust vulnerability seismic-capacity predictions for earthquakedamaged structures, error-domain model falsification (EDMF) is used in this paper. EDMF combines multi-sourced biased uncertainties and is not sensitive to estimates of error correlation that undermines traditional applications of model-based data interpretation (Pasquier \& Smith, 2015a). Correlations between errors related to multiple predictions cannot be known precisely and assuming no correlation between errors is unsafe. By avoiding assumptions regarding correlations between predictions, EDMF sacrifices precision (defined as the range of uncertainty in predictions) in order to improve accuracy (defined as the probability of including the true, or accurate, prediction in the predicted range). Thus, using the EDMF methodology no most likely (or optimal) parameter value and consequently no most-likely prediction is provided. Also, EDMF results in uniform probabilities of candidate physical models, rather than informed probability distributions (such as Gaussian distributions) that point to most likely estimates of parameter values. Through tens of full-scale applications in several countries, EDMF has been observed to be more easily understandable than Bayesian updating by the practicing engineers who must combine measurement data with observations (Smith, 2016).

The model falsification approach builds upon the strategy that measurement data should ideally be used to falsify inappropriate model instances instead of validating or optimising single models (Robert-Nicoud, Raphael, \& Smith, 2005). This concept, intuitively understood by engineers, is based on the principles of scientific discovery (Popper, 1959). Parametric uncertainty of physics-based models is accommodated through discrete populations of models, which represent a sample of parameter combinations and are used to simulate structural behaviour (Raphael \& Smith, 1998). Goulet and Smith (2013) have formalised the concept of model falsification into a data- 
interpretation technique, EDMF, that accommodates systematic and biased modelling uncertainties in addition to (generally white-noise) measurement uncertainties. EDMF has been shown to be more accurate than traditional applications of Bayesian model updating for prognosis tasks that involve extrapolation of linear elastic models (Pai, Nussbaumer, \& Smith, 2018; Pasquier \& Smith, 2015a) as well as nonlinear models (Reuland et al., 2017). Important decisions in the field of asset management (such as remaining-life calculation, repair, extension and improvement through retrofit) require extrapolation of behaviour models beyond the scope of measurements.

The cornerstone of EDMF is the comparison of model predictions with measured behaviour. An initial population of model instances is obtained from sampling the parameter space. The parameter space is defined using engineering heuristics to define bounds to the $n_{\theta}$-dimensional parameter space $\boldsymbol{\theta}=\left\{\theta_{1}, \ldots, \theta_{n_{\theta}}\right\}$. A physics-based model $\mathbf{g}(\cdot)$ predicts the structural response $g_{i}(\boldsymbol{\theta})$ corresponding to the $n_{m}$ measured quantities $y_{i}$. However, due to inevitable simplifications, omissions and idealisations characterising any physical model $\mathbf{g}(\cdot)$, the prediction of a model instance, $g_{i}(\boldsymbol{\theta})$, is complicated by model errors $u_{g}$. Similarly, the measurement, $\mathbf{y}$, is prone to a measurement error $u_{y}$. According to Eq. 1, the sum of the model prediction based on the correct, yet unknown, parameter values $\boldsymbol{\theta}^{*}$ and the model error equals the sum of the measurement and the measurement error. Both sides of Eq. 1 equal the true, yet unknown, behaviour of the system.

$$
\forall i \in\left\{1, \ldots, n_{m}\right\}: g_{i}\left(\boldsymbol{\theta}^{*}\right)+u_{i, g}=y_{i}+u_{i, y}
$$

In complex open-world applications, such as civil engineering structures, neither measurement error nor modelling error is known precisely. Nevertheless, heuristic knowledge is used to estimate ranges of uncertainty. Generally, engineering experience and knowledge lead to conservative estimates of the uncertainty related to model and measurement errors. Heuristic estimates of model and measurement uncertainties usually take the form of bounds. In a few cases when more information is available, probability distributions that are more elaborate than the uniform distribution are estimated. Modelling and measurement error are thus described by the random variables $U_{g}$ and $U_{y}$. Estimating uncertainties contains subjective assessment and may differ between engineers. However, EDMF has been shown in the past to be robust with respect to uncertainty misevaluations Reuland et al. (2017). In addition, sensitivity analyses with respect to estimated uncertainty bounds should be performed.

By combining modelling and measurement uncertainties, the joint probability density function $(\mathrm{PDF})$ of the estimated total error uncertainty $f_{U}$ is obtained according to Eq. 2. As a result of the high levels of uncertainty that are associated with models of complex civil-engineering structures, the joint error pdf $f_{U}$ is generally dominated by modelling uncertainty. The joint uncertainty $\mathrm{PDF} f_{U}$ is subsequently used to define thresholds, $T$. Based on these thresholds, model instances are falsified.

$$
f_{U} \sim U_{y}-U_{g}
$$

The threshold values $T_{l o w, i}$ and $T_{h i g h, i}$ delimit the shortest interval that assures a cumulated probability corresponding to a target reliability $\phi_{d}$ (see Eq. 3). The target reliability $\phi_{d}$ is pre-defined and is related to the probability $\left(1-\phi_{d}\right)$ that the right parameter combination is falsely discarded. Fixing such a target is a common starting 
point for engineers; many code provisions, from safety factors to fatigue strength curves, are fixed using pre-defined target reliabilities. When the uncertainty distribution $f_{U}$ exactly defines the true error distribution, the probability of including the correct parameter combination in the model set is given by the value of $\phi_{d}$. The number of measurements $n_{m}$ is included in Eq. 3 because in most applications, more than one measured quantity from one sensor location is available. To account for the simultaneous comparisons of $n_{m}$ measured quantities with model predictions, the Šidák correction is employed for multiple hypothesis testing (Šidák, 1967) when calculating threshold bounds.

$$
\forall i \in\left\{1,2, \ldots, n_{m}\right\}: \phi_{d}^{1 / n_{m}}=\int_{T_{\text {low }, i}}^{T_{\text {high }, i}} f_{U_{i}}\left(u_{i}\right) d u_{i}
$$

Models that predict residuals between model predictions and measured values that fall inside the falsification thresholds bounds for all $n_{m}$ measured quantities are candidate models, $\boldsymbol{\theta}^{\prime \prime}$, according to Eq. 4 . The combined uncertainty distribution includes heuristic estimations. Forms of the uncertainty distributions as well as correlations between the $n_{m}$ quantities are unknown. In such contexts, all candidate models are considered to be equally likely. The finite number of uncertainty sources undermines the applicability of the central limit theorem in such cases (Pasquier \& Smith, 2015b). A graphical summary of error-domain model falsification for one measured quantity $i$ is provided in Fig. 1.

$$
\forall i \in\left\{1,2, \ldots, n_{m}\right\}: T_{\text {low }, i} \leq g_{i}\left(\boldsymbol{\theta}^{\prime \prime}\right)-\hat{y}_{i} \leq T_{\text {high }, i}
$$

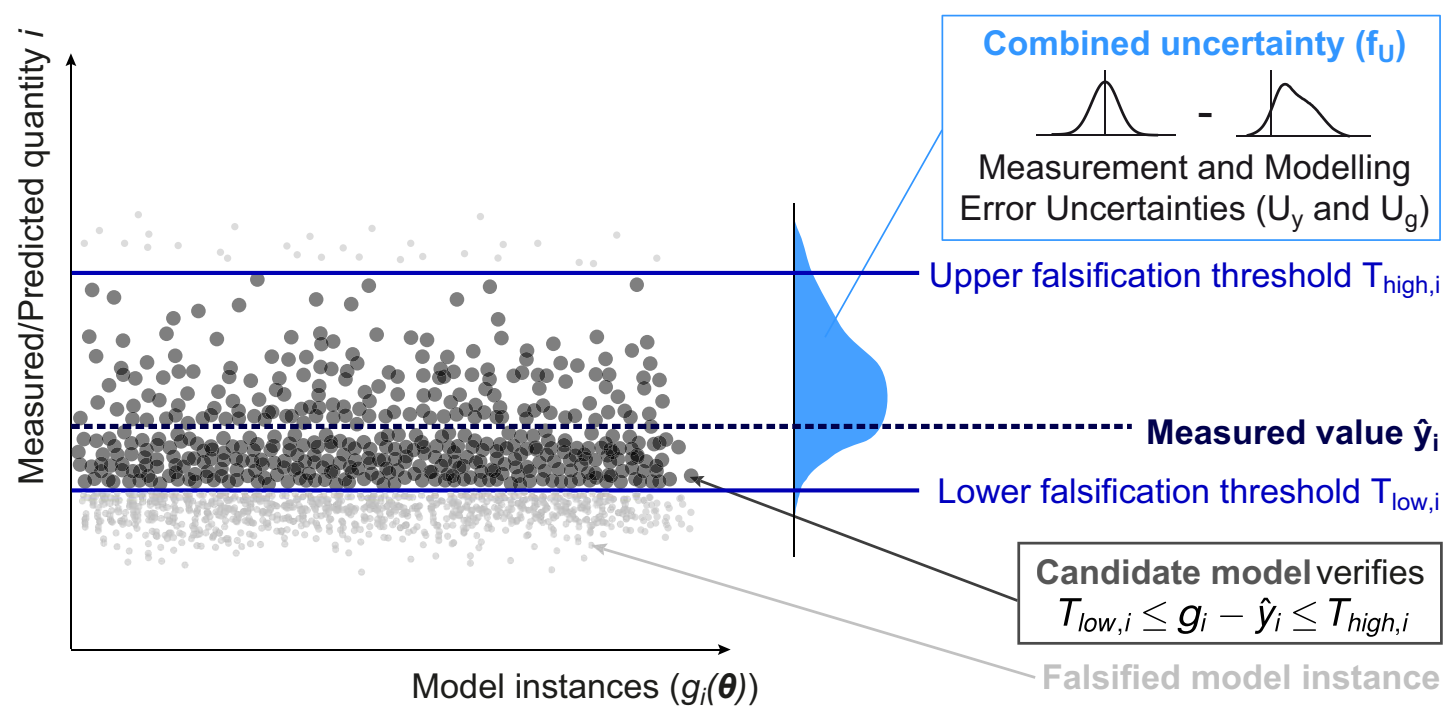

Figure 1. Illustration of the error-domain model-falsification methodology. Falsification thresholds are derived from combined uncertainty. Candidate models fall between falsification thresholds for all measured quantities. Once identified, candidate models are taken to be equally likely because knowledge of informed uncertainty distributions is poor. 


\subsection{Combining visual inspection and modal measurements to identify earthquake-damaged structures (diagnosis)}

A general framework for structural identification of earthquake-damaged buildings is proposed, see Fig. 2. Assessing the residual capacity of a building starts with structural diagnosis. The diagnosis task is an inverse problem that is, by definition, ambiguous; many causes (model instances) can produce the same effect (measurements) within a margin of uncertainty. EDMF explicitly accommodates such inherent ambiguity of inverse problems, and therefore it is well-suited to assess earthquake-damaged buildings.

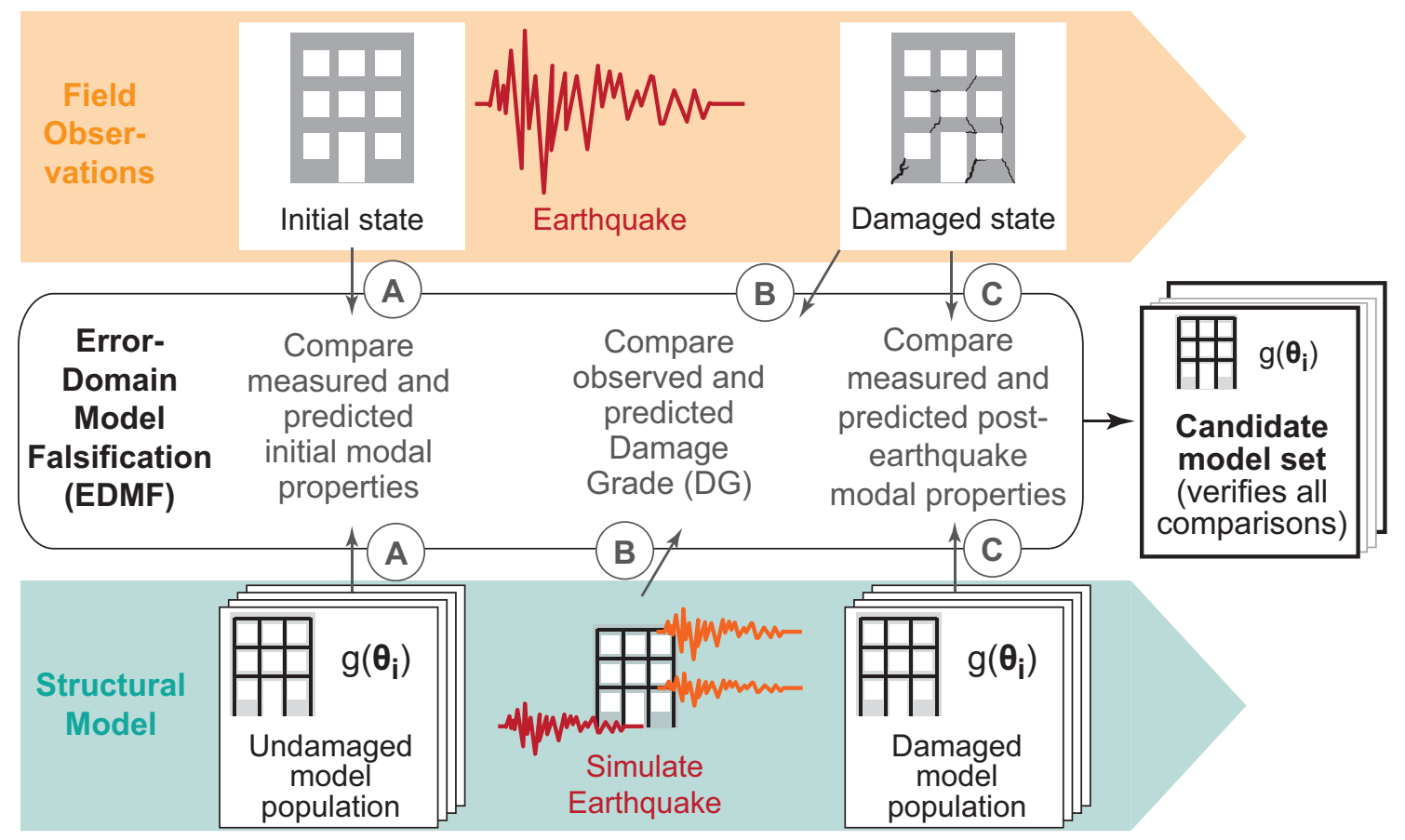

Figure 2. Framework for measurement-based structural-identification methodology for earthquake-damaged buildings. From a population of models, observations and measurements are used to identify a candidate model set containing appropriate behaviour models for prediction, see Fig. 4. Data flows A, B and C indicate data interpretation events.

The proposed assessment of the residual capacity of earthquake-damaged buildings relies on a non-linear hysteretic model to link the initial building state with the postearthquake state. This model simulates the structural response of a building to an earthquake excitation. In addition, structural quantities, e.g. peak displacements at the top of the building and forces at the base, experienced by the structure during an earthquake can be derived from the simulation. Non-linear hysteretic models involve two type of parameters: linear elastic parameters (such as Young's modulus) and non-linear parameters (such as material strength).

In order to reduce the parametric uncertainty of the non-linear hysteretic model, quantities predicted by the model are compared with measurements of behaviour of the real structure. This paper proposes two types of measurements to falsify model instances. These are i) modal properties of the structures representative of the two building states, before and after the earthquake and ii) the damage grade (DG) observed by visual inspection, see Fig. 2 .

Although modal parameters of the building before and after the earthquake provide engineers with objective data, such comparisons are not intended to replace visual 
inspection. Instead, these additional data sources are meant to support and complement visual information from visual inspection. Visual inspection remains valuable and therefore, it is an essential part of the post-earthquake assessment framework, which is presented in this paper. In addition, visual inspection is needed to verify collapse mechanisms that are not covered by structural models, such as out-of-plane mechanisms as well as damage to non-structural elements that are potentially dangerous (such as collapse of a chimney).

The DG that is observed through visual inspection is transformed to a physical quantity in order to enable model falsification. A link between the damage grade and the maximum displacement, which is sustained by a structure during a seismic event, has been established in the past (Calvi et al., 2006; Lagomarsino \& Giovinazzi, 2006). The boundaries $S_{d, i}$ (with $i=1: 4$ ) between the five DGs that have been defined by the European Macroseismic Scale (EMS98) are defined through limit-state mechanical properties of the structure (Grünthal, Musson, Schwarz, \& Stucchi, 2001), as recalled in Equation 5. $D_{y}$ and $D_{u}$ are respectively yield and ultimate displacement of the structure. Yield and ultimate displacement depend on parameter values of the structural model and may be derived either from a push-over curve for sophisticated models or directly form model parameters for simplified models (such as bilinear single-degree-of-freedom representations).

$$
\left\{\begin{array}{c}
S_{d, 1}=0.7 \cdot D_{y} \\
S_{d, 2}=1.5 \cdot D_{y} \\
S_{d, 3}=0.5 \cdot\left(D_{y}+D_{u}\right) \\
S_{d, 4}=D_{u}
\end{array}\right.
$$

By tightly integrating visual-inspection outcomes into the proposed framework, the observed DGs are used to falsify model instances using EDMF. Two types of uncertainties arise when DGs are used as data source for falsification. First, the definitions of boundaries separating the DGs are based on assumptions and empirical observations. In addition, visual inspection (although it is conducted by experts and strongly formalized) may lead to observed DGs that differ from the definition in Equation 5. Uncertainty related to the definition of DG boundaries and the potential error in an assessed DG form the uncertainty distribution $\left(\epsilon_{D G b o u n d}\right)$. As stated before, the bounds of this uncertainty have to be derived from engineering heuristics. In absence of precise information, a conservative range of $\pm 50 \%$ is proposed for the damage boundary definition value. Observations from past post-seismic building assessments may help to derive more precise uncertainty ranges in the future. In addition to DG defintions, various sources of model uncertainty are associated with maximumdisplacement predictions. These uncertainties, $\epsilon_{\text {model }}$, depend on the selected model and need to be estimated using engineering judgement. Since exact values of the error cannot be known, a conservative estimate of the model error should be provided by the engineer.

The quantification of an observed DG results in intervals for the maximum displacement. Thus, the equation of model falsification (Eq. 4) is adapted to accommodate a measured interval instead of a single measured value. Yield and ultimate displacement of the structure are unknown parameters and therefore the boundaries between the DGs $S_{d, i}(\boldsymbol{\theta})$ depend on the model instance. Thus, for an observed DG $j$, Eq. 6 gives the condition for the predicted maximum displacement $d_{\max }(\boldsymbol{\theta})$ to be retained in the candidate model set. 


$$
S_{d, j-1}(\boldsymbol{\theta})+\varepsilon_{\text {DGbound }} \leq d_{\text {max }}(\boldsymbol{\theta})+\varepsilon_{\text {model }} \leq S_{d, j}(\boldsymbol{\theta})+\varepsilon_{D G b o u n d}
$$

Similarly to Eq. 3, threshold values, $T_{l o w, D G}$ and $T_{\text {high,DG}}$, on the total error uncertainty distribution of $U_{D G}=\epsilon_{D G b o u n d}-\epsilon_{\text {model }}$ are calculated and subsequently used to falsify model instances as presented in Eq. 7. A graphical summary of model falsification based on observed $D G$ s is provided in Fig. 3 .

$$
\left\{\begin{array}{c}
d_{\max }(\boldsymbol{\theta})-S_{d, j-1}(\boldsymbol{\theta}) \geq T_{\text {low }, D G} \\
d_{\max }(\boldsymbol{\theta})-S_{d, j}(\boldsymbol{\theta}) \leq T_{h i g h, D G}
\end{array}\right.
$$

The definition of the boundaries bounding $D G 1$ and $D G 2$ are independent of the ultimate displacement $D_{u}$. Thus, no reduction of the parameter uncertainty related to the ultimate displacement $D_{u}$ can be achieved for observed DGs lower than $D G 3$.

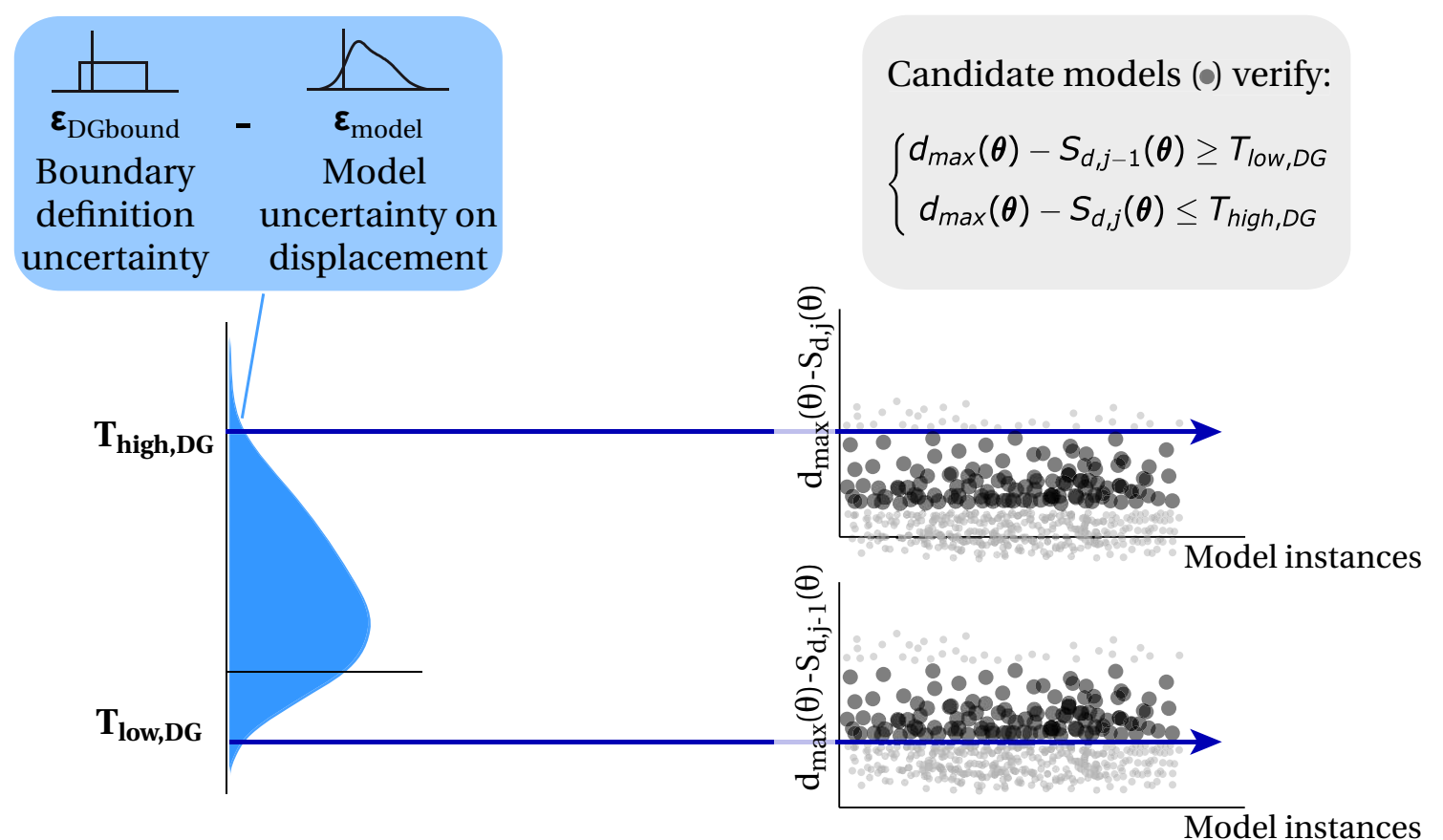

Figure 3. Illustration of the methodology of model falsification using the observed damage grade (DG). For an observed DG $\mathrm{j}$ the lower threshold depends on the lower bound, $\left(S_{d, j-1}\right.$, of the range attributed to DG $\mathrm{j}$ and the upper threshold depends on the higher bound, $S_{d, j}$, of DG j.

\subsection{Vulnerability prognosis for earthquake-damaged structures}

Although identifying structural properties of a building after an earthquake is an important step, it is not the final objective of post-earthquake building assessment. The objective of the proposed methodology is to assess the residual capacity of a building to withstand future earthquake actions. Thus, the candidate model set that has been identified through EDMF is used to predict the structural response of a damaged building under future earthquakes of varying intensities. 
Predicting the response of a damaged building to further seismic excitation is a forward problem. As ambient-vibration measurements are used and earthquake actions are simulated, model predictions involve extrapolation (for example, loading differs from the measurement conditions). Structural behaviour during aftershocks or future earthquakes differs significantly from measurement configurations (i.e. ambient vibrations). Thus, inaccurate estimations of total prediction uncertainties can lead to biased and potentially unsafe extrapolations (Pasquier \& Smith, 2015a).

Uncertainties governing seismic displacement predictions largely differ from identification uncertainties, which are related to modal properties derived from ambient vibrations. Therefore, prediction uncertainties, $U_{q}$, need to be re-evaluated and may differ from identification uncertainties, $U_{g}$ (see Eq. 2). The behaviour prediction, referred to by the random variable $q$, are obtained by combining the remaining parametric uncertainty in the candidate model set (see Eq. 4) with the model-prediction uncertainty $U_{q}$, as shown in Eq. 8. A Monte-Carlo combination scheme is implemented for uncertainty combination.

$$
q=g(\boldsymbol{\theta})+U_{q}
$$

Since prediction is a forward problem, model uncertainties are added to the predicted values, as opposed to identification uncertainties that are subtracted from the combined uncertainty (as identification is an inverse problem, see Eq. 2). For instance, a model that is considered to overestimate displacement (identification uncertainty, $u_{g}$ in Eq. 1 , is biased in the negative direction) leads to falsification thresholds that are biased towards higher displacement values. The prediction uncertainty of such a model, which is added to the predicted displacement values, presents a bias towards lower displacement values.

After estimating prediction uncertainties, a bounded prediction interval can be calculated involving a similar procedure than to derive identification thresholds, see Eq. 3 . Therefore, decision makers define a target probability $\phi_{p}$ that is used to calculate the prediction interval. The probability that the right model prediction is included in the prediction range has a lower bound $\phi=\phi_{p} \cdot \phi_{d}$. This lower bound holds true if the uncertainty is correctly estimated (Pasquier \& Smith, 2016).

A common approach for estimating the vulnerability of an earthquake-damaged building involves determination of the probability of the building exceeding EMS98 DGs for a given earthquake level. The prediction uncertainty and the uncertainty of DG definitions are combined through a Monte-Carlo sampling process with $n_{s p}$ samples. A sufficient number of samples $\left(n_{s p}\right)$ is used to guarantee stable distributions for the combined prediction uncertainty. Fig. 4 summarizes the process of uncertainty combination for structural vulnerability prognosis using a candidate model set resulting from model falsification.

\section{Case study - shake-table tests on a mixed concrete masonry building at half-scale}

A mixed reinforced-concrete unreinforced-masonry building (Fig. 5) is analysed to illustrate the framework, which is proposed in Fig. 2. The four-story building has been tested at half-scale on a shake table by Beyer, Tondelli, Petry, and Peloso (2015). Multiple shake-table sequences characterized by increasing amplitudes were tested and 


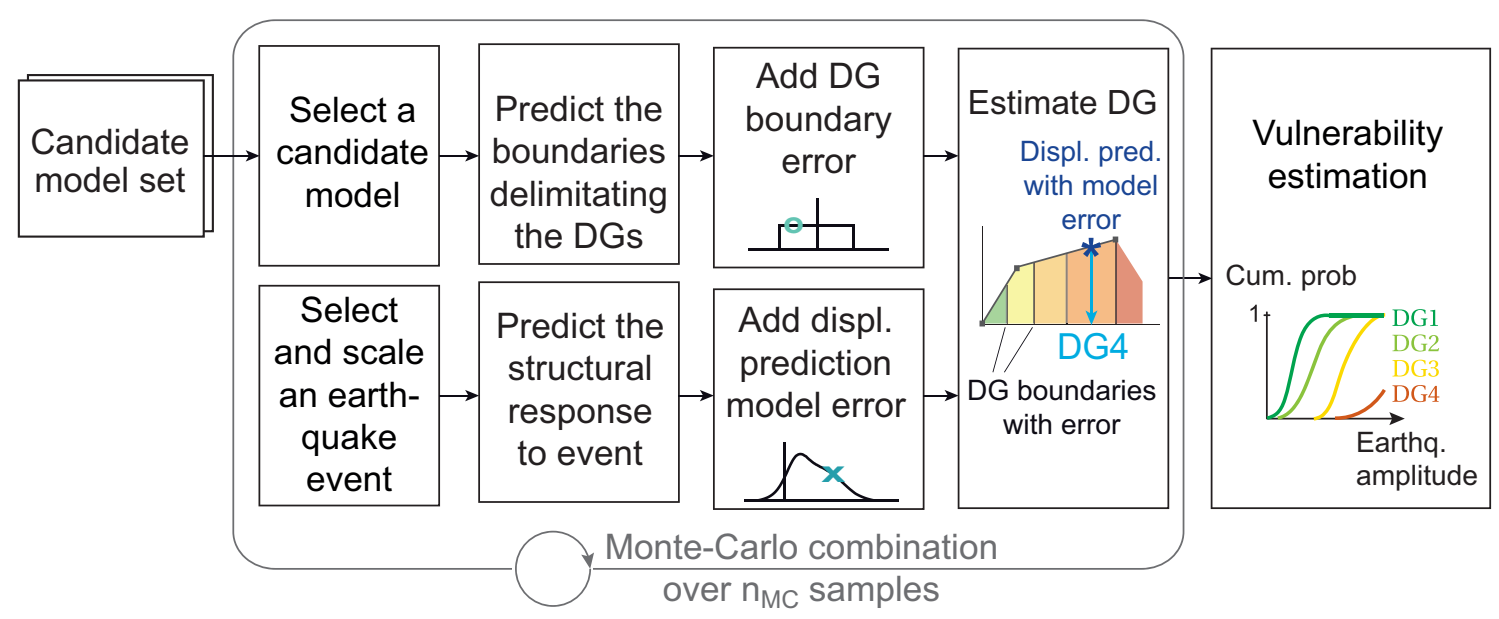

Figure 4. Methodology for vulnerability prognosis using the identified candidate model set. A Monte-Carlo uncertainty combination is performed involving a randomly selected candidate model with a randomly selected earthquake event (defined by a ground-motion signal and a shaking amplitude). Displacement uncertainty is added to the displacement demand of the selected candidate model under the selected earthquake. DGboundary uncertainty is added to the bounds delimiting the DGs derived from the parameter values of the selected candidate model. The derived DG for the selected candidate model, earthquake signal and uncertainty realisations is stored and the process is repeated over a defined number of Monte-Carlo samples.

between each run, ambient vibrations were simulated. The peak ground acceleration (PGA) characterizing each test run has been measured. The input motion applied by the shake table is the east-west component measured at the ground station of the Ulcinj-Hotel Albatros station during the April 15th, 1979 Montenegro earthquake. This accelerogram has been scaled to increasing PGAs for the shaking-table tests. The evolution of natural frequencies and DGs under shaking tests with increasing PGAs is reported in Table 1. A gradual reduction in the natural frequencies with cumulating damage can be observed. Stiffness recovery that can be observed after shaking test 5 can be explained by the low amplitude of white-noise (WN) excitation (order of magnitude lower than for all other WN tests). A detailed description of the shaking test observations and illustrations of the deteriorated specimen can be found in Beyer et al. (2015).

As revealed by tests on mixed unreinforced-masonry reinforced-concrete structures, combining both materials results in a better vertical distribution of damage (Paparo \& Beyer, 2013). In addition, strength of combined units increases until failure of the unreinforced-masonry walls. Therefore, combined structures have been found to show no strength degradation (Paparo \& Beyer, 2014). Finally, an important aspect of the tested specimen is the smooth stiffness degradation. This smooth stiffness degradation is reflected by the gradual reduction in natural frequencies (see Table 1) that results from multiple tests with increasing amplitudes of shaking.

The use of a laboratory specimen for illustration does not necessarily reflect realistic conditions for post-earthquake assessment. The idealized boundary conditions and the absence of secondary elements considerably reduce uncertainty when comparing the real structure with the physics-based model. In addition, pseudo-ambient vibration conditions, involving higher acceleration amplitudes and perfect white noise, increase the quality of measurements. However, no case-studies on real structures that have been measured before and after an earthquake to enable a comparison of behaviour prognosis with real behaviour are available for building types that compare to existing 


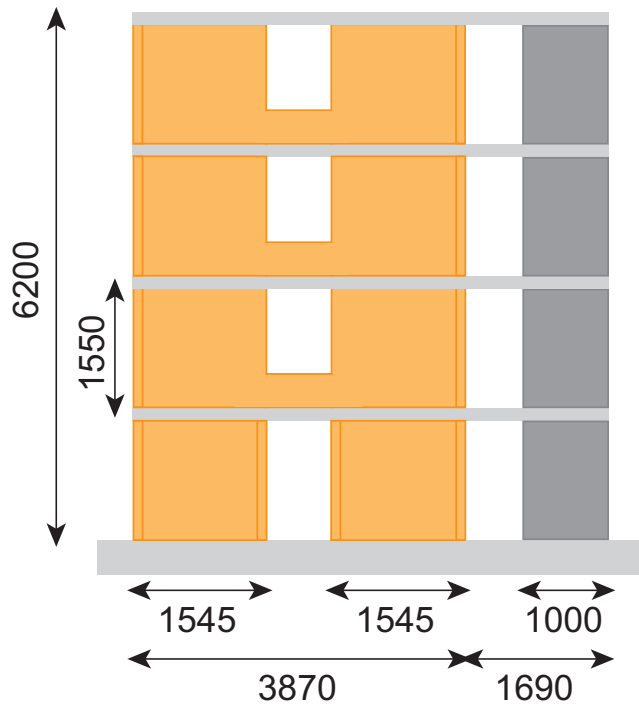

Elevation

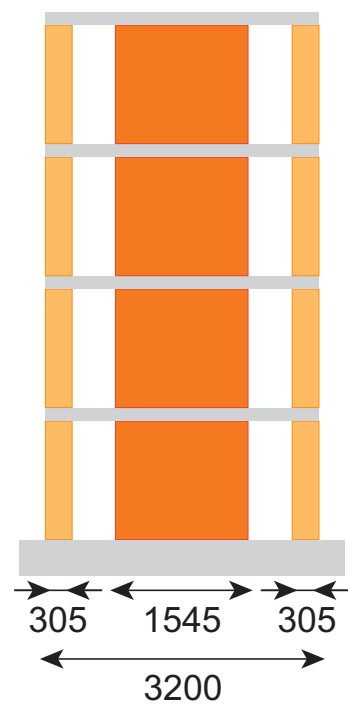

Section

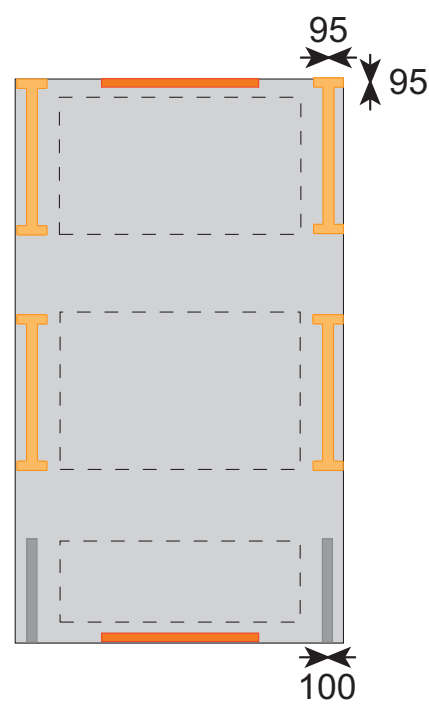

Top view

Masonry walls (loaded in plane)

Masonry walls (loaded out of plane) Reinforced concrete walls $\square$ Reinforced concrete slabs $\left\llcorner_{-} \_\right.$Additional masses (similarity laws)

Figure 5. Description of the half-scale mixed unreinforced-masonry reinforced-concrete laboratory specimen tested on a shaking table by Beyer et al. (2015). Dimensions are given in millimetres.

Table 1. Evolution of natural frequencies due to cumulative damage of the specimen. Smooth stiffness degradation is observed as natural frequencies are reduced gradually. WN refers to white-noise tests performed after the shaking test to derive natural frequencies.

\begin{tabular}{ccccccc}
\hline Test & $\begin{array}{c}f_{1} \\
{[\mathrm{~Hz}]}\end{array}$ & $\begin{array}{c}\text { Reduction } f_{1} \\
{[\%]}\end{array}$ & $\begin{array}{c}f_{2} \\
{[\mathrm{~Hz}]}\end{array}$ & $\begin{array}{c}\text { WN level } \\
{\left[\mathrm{mm} / \mathrm{s}^{2}\right]}\end{array}$ & DG & $\begin{array}{c}\text { PGA } \\
{\left[\mathrm{m} / \mathrm{s}^{2}\right]}\end{array}$ \\
\hline 3 & 6.6 & -16.5 & 24.1 & 176 & 1 & 2.1 \\
4 & 5.8 & -26.6 & 23.8 & 176 & 2 & 3.4 \\
5 & 6.3 & -20.3 & 23.9 & 26.2 & 2 & 3.9 \\
7 & 5.3 & -33 & 23.2 & 175 & 2 & 7.5 \\
8 & 5 & -37 & 21.6 & 176 & 3 & 6.3 \\
9 & - & - & - & - & $4-5$ & 14.7 \\
\hline
\end{tabular}


buildings in Europe. Consequently, this study provides an upper-bound performance feasibility indicator for the proposed methodology.

\subsection{Identification scenarios}

The building state after the sixth shaking event is evaluated. Progressive deterioration of the specimen due to multiple previous shaking events are accounted for by simulating all the six previous shakes. The maximum PGA during the first six shake tests is 7.5 $\mathrm{m} / \mathrm{s}^{2}$. The initial fundamental frequency is $f_{0}=7.8 \mathrm{~Hz}$ and the fundamental frequency measured after the sixth shaking is $f_{p e}=5.3 \mathrm{~Hz}$. Visual inspection performed after the test reported a $D G_{V I}=2$.

Five scenarios are defined by availability of measurements. The scenarios are chosen to represent realistic situations of post-earthquake assessment. Scenarios $S_{1}$ and $S_{2}$ involve a single measurement source, either $f_{p e}$ (data interpretation event $\mathrm{C}$ in Fig. 2) or $D G_{V I}$ (data interpretation event B in Fig. 2). Scenarios $S_{3}$ and $S_{4}$ involve two data sources, either $f_{p e}$ and $D G_{V I}$ or the fundamental frequencies before (data interpretation event A in Fig. 2) and after the earthquake $\left(f_{0}\right.$ and $\left.f_{p e}\right)$. Finally Scenario $S_{5}$ includes the assumption that all three sources of measurements are at disposal. The five identification scenarios are summarized in Table 2.

Table 2. Summary of scenarios regarding available measurement sources

\begin{tabular}{lc}
\hline Scenario & Measurement sources for identification \\
\hline$S_{1}$ & Post-earthquake frequency only \\
$S_{2}$ & Post-earthquake visual inspection only \\
$S_{3}$ & Post-earthquake frequency and inspection \\
$S_{4}$ & Frequencies before and after earthquake \\
$S_{5}$ & Frequencies before/after earthquake and visual inspection \\
\hline
\end{tabular}

\subsection{Structural model}

A single-degree-of-freedom (SDOF) model is used to model the test structure. SDOF models involve the assumption that the behavior of a structure, as well as the structural damage, is governed mostly be the fundamental mode. Given the vertical distribution of damage and the smooth and gradual stiffness reduction this assumption is deemed acceptable for the tested structure. In addition, the modal mass of the first mode exceeds $75 \%$ of the total mass. Finally, the symmetrical design and the unilateral shaking strongly reduce influence of torsional effects structural damage.

Nonlinear structural behavior is modelled using a bilinear law, which is characterised by initial stiffness, $k_{0}$, yield force, $V_{R}$, ultimate displacement, $D_{u} l t$, and post-yield hardening, $\operatorname{hard}_{p y}$ (defined as a percentage of the initial stiffness). The hysteretic behaviour relationship that is used to predict the structural behaviour during an earthquake is the modified Takeda relationship (Takeda, Sozen, \& Nielsen, 1970). Two parameters govern unloading $(\alpha)$ and reloading $(\beta)$ behaviour in the Takeda model. Finally, an overall damping ratio, $z$, is defined.

The initial fundamental frequency, $f_{0}$, depends on the initial stiffness, $k_{0}$, and the modal mass, $m_{i}$, of the first mode, as defined in Eq. 9. The post-earthquake fundamental frequency, $f_{p e}$, is defined based on the secant stiffness to the reloading point that is principally governed by the maximum displacement, $d_{\max }$, that the structure undergoes during an earthquake sequence and stiffness degradation for reloading, $\beta$ (Eq. 10). 


$$
\begin{gathered}
f_{0}=\frac{\sqrt{k_{0} / m_{1}}}{2 \pi} \\
f_{p e}=\left\{\begin{array}{cc}
\frac{1}{2 \pi} \sqrt{\frac{D_{y} \cdot k_{0}+k_{p y} \cdot k_{0}\left(d_{\max }-D_{y}\right)}{D_{y}+(1-\beta) \cdot\left(d_{\max }-D_{y}\right)} \cdot \frac{1}{m_{1}}} & \text {, if } d_{\max }>D_{y} \\
f_{0} & \text {,otherwise }
\end{array}\right.
\end{gathered}
$$

Relying on a SDOF model involves the assumption that structural behaviour during an earthquake is mostly governed by the first mode. Thus the SDOF model that is used to simulate the hysteretic response of the building is defined by the fundamental mode. A sensitivity analysis is carried out to define the primary parameters, which have a relative importance superior to five percent for one of the predicted quantities.

The dynamic parameters of the intact building are derived from a lumped-mass model of the test structure. Young's moduli of reinforced concrete $\left(20-35 \mathrm{kN} / \mathrm{mm}^{2}\right)$ and of in-plane-loaded masonry $\left(4-12 \mathrm{kN} / \mathrm{mm}^{2}\right)$ are parameters of the lumped-mass model as well as well as density of reinforced concrete $\left(2.3-2.5 \mathrm{t} / \mathrm{m}^{3}\right)$ and masonry $(1.4$ to $\left.1.8 \mathrm{t} / \mathrm{m}^{3}\right)$ ). No geometric uncertainty is considered in this application.

A sensitivity analysis is carried out in order to identify the parameters that have a significant contribution (exceeding 5\%) on predicted values (frequency and maximum displacement). Based on the sensitivity analysis, three parameters are discarded from the primary parameter set: densities of reinforced concrete and masonry and the $\alpha$ parameter governing unloading in the Takeda model. The remaining seven primary parameters are summarized in Table 3 (parameters $\theta_{1}-\theta_{7}$ ) with the ranges of parameter values and the number of divisions for grid sampling.

Thus, the initial model population for hysteretic nonlinear time-history analyses contains 8640 model instances that are obtained from grid-sampling the parameter space. Ultimate drift capacity of the structure influences the DG predictions, however it cannot be identified based on an observed $D G 2$ and it does not influence the nonlinear simulations.

Table 3. Primary parameters for identification with the corresponding initial ranges and divisions. The initial model population contains 6912 model instances.

\begin{tabular}{lcccc}
\hline Parameter & Description & Unit & Range & Divisions \\
\hline$\theta_{1}$ & Young's modulus Concrete & $\mathrm{kN} / \mathrm{mm}^{2}$ & {$[20,35]$} & 4 \\
$\theta_{2}$ & Young's modulus Masonry & $\mathrm{kN} / \mathrm{mm}^{2}$ & {$[4,12]$} & 5 \\
$\theta_{3}$ & Yield limit $\left(V_{R}\right)$ & $\mathrm{kN}$ & {$[100,1100]$} & 6 \\
$\theta_{4}$ & Damping ratio $(z)$ & $\%$ & {$[2.5,7.5]$} & 3 \\
$\theta_{5}$ & Post-yield hardening (hard $\left.{ }_{p e}\right)$ & $\%$ & {$[0,15]$} & 6 \\
$\theta_{6}$ & Reloading parameter $(\beta)$ & - & {$[0,0.6]$} & 4 \\
$\theta_{7}$ & Ultimate drift $\left(D_{u l t}\right)$ & $\%$ & {$[0.4,1.2]$} & 5 \\
$\theta_{8}$ & Earthquake amplitude (PGA) & $\%$ & {$[50,200]$} & 4 \\
$\theta_{9}$ & Earthquake Signal & - & - & 13 \\
\hline
\end{tabular}

\subsection{Sources of uncertainties}

Uncertainties resulting from multiple error sources are assessed for the shake-table case study. A measurement uncertainty, associated to measurement precision and to time-domain to frequency-domain transformation, is estimated to be a native Gaussian 
zero-mean distribution with a standard deviation of $0.2 \mathrm{~Hz}$. This is compatible with previous analyses (Goulet, Michel, \& Smith, 2013).

Several sources of modelling uncertainties are estimated. Due to poor knowledge of uncertainty distributions, all sources of model uncertainties are assumed to follow uniform (uninformed) distributions. The error sources affect the predicted fundamental frequencies as well as the predicted maximum displacements during the earthquake. However, as uncertainties mainly affect structural stiffness, uncertainties may be higher for displacement than for frequencies. Relative uncertainties are defined according to Eq. 11, therefore negative values relate to an overestimation of a physical quantity by the chosen model.

$$
\epsilon_{g}=\frac{\text { Truth }- \text { Model }}{\text { Truth }}
$$

Ambient vibrations are used to derive the fundamental frequency. However, such ambient excitation sources are characterized by very low amplitudes. A bilinear forcedisplacement relationship is unable to reproduce the aforementioned higher stiffness at low amplitudes of excitation. Thus, the model is estimated to underestimate the natural frequency derived from ambient vibrations by a maximum of $15 \%$. In applications involving real buildings, this error is considerably higher due to the contribution of non-structural elements and to the even lower levels of excitation.

A lumped-mass model of the building that is used to predict initial frequencies. Such a model idealizes boundary conditions and connections between structural elements to be perfectly fixed. In addition, the slab is assumed to be rigid in the horizontal direction. Based on the preceding considerations, modelling uncertainties resulting from the lumped mass model are considered to be biased towards stiffness overestimation. Given the simplified nature of the model, engineering heuristics lead to an estimate of a minimum uncertainty bound of $20 \%$ on predicted stiffness values. Therefore, modelling uncertainty is taken to be bounded between -20 and +10 percent for the natural frequency (depending on the square-root of stiffness) and -20 and +40 percent for the maximum displacement. Uncertainties on displacement is evaluated to be higher, as displacement linearly depends on stiffness. The structural response to earthquake actions is simulated using SDOF models. In addition to the error introduced by relying to lumped-mass models, an SDOF representation overestimates displacements when compared to MDOF representations (Lestuzzi, Belmouden, \& Trueb, 2007). Therefore, a displacement uncertainty ranging from $-30 \%$ to $+20 \%$ is assumed.

The prediction of the frequency reduction due to post-yield displacements (damage) involves estimating the damaged stiffness. A simplified (and conservative) approach consists of using the secant stiffness to the maximum displacement (see Eq. 10). This simplification may lead to an underestimation of post-earthquake frequencies. Indeed, under vibrations with low amplitudes, structures may regain higher stiffness values after the shaking event (Michel et al., 2011). In order to accommodate this error, an uncertainty range between 0 and 10 percent is estimated. This uncertainty is exclusively positive because the conservative approach cannot lead to an underestimation of stiffness.

Finally, the uncertainty related to the DG definition from the maximum displacement is taken into account through a relative error of $\mp 50 \%$ on the boundary value $\left(S_{d, i}\right)$. This means for instance that the boundary between DG2 and DG3, defined to be 1.5 times Dy is described as a uniform distribution bounded by the interval $\left[0.75 \cdot D_{y}, 2.25 \cdot D_{y}\right]$. 
Table 4. Estimates of the ranges of modelling-uncertainty sources.

\begin{tabular}{lccc}
\hline Source & Nature & Applies to & Error bounds \\
\hline Ambient vibrations & Uniform, Relative & Natural frequency & {$[0 \%, 15 \%]$} \\
Structural model & Uniform, Relative & Natural frequency & {$[-20 \%, 10 \%]$} \\
& & Displacement & {$[-20 \%,+40 \%]$} \\
Stiffness updating & Uniform, Relative & Natural frequency & {$[0 \%,+10 \%]$} \\
SDOF approximation & Uniform, Relative & Displacement & {$[-30 \%,+20 \%]$} \\
DG definition & Uniform, Relative & DG boundaries & {$[-0.5,0.5] \cdot D_{y}$} \\
\hline
\end{tabular}

In absence of additional information, such an approach leads to conservative results. Table 4 summarizes the estimated uncertainty distributions related to the various error sources.

In addition to the epistemic sources of modelling uncertainty described above, three parameters add to the combined uncertainty distribution. Such parameters, which have no significant influence on simulated structural behaviour are considered to be secondary parameters. The secondary parameters are respectively density of masonry and concrete as well as the $\alpha$-parameter governing unloading in the modified Takeda hysteretic law (see Section 3.2). The numerical influence of the three secondary parameters is simulated using 2000 Monte-Carlo samples and treated as supplementary source of uncertainty. The relative importance of the secondary parameters depends on the intensity of the simulated earthquake, however in all cases the influence remains negligible compared with the model uncertainties (less than $4 \%$ of the combined uncertainty values).

\subsubsection{Taking into account ground-motion uncertainty}

The uncertainty related to the ground motion is taken into account through adding two parameters that are related to the signal and intensity of the earthquake. If no direct measurement of the sustained earthquake is available, multiple earthquake signals are simulated to reflect accelerogram uncertainty. In the present case study, 12 accelerograms are randomly selected from the European Strong Motion Database (Ambraseys et al., 2004) under the constraint to provide a large variety of earthquake characteristics, such as epicentral distances, soil conditions and magnitudes. The structural response to these 12 accelerograms is simulated in addition to the true shake-table input signal. Fig. 6 shows the characteristics of the 12 selected acceleration signals: response spectra (a) as well as magnitude and epicentral distance (b). All the acceleration spectra (see Fig. 6,a) are normalized with respect to the peak ground acceleration (PGA). In total, 13 input signals scaled to four PGA values (ranging from 50 to 200 percent of the true PGA) are simulated (see earthquake-signal-related parameters $\theta_{8}$ and $\theta_{9}$ in Table 3 ). Thus, in total 52 earthquake signals are simulated for each model instance (13 ground-motion signals and 4 PGA scalings). The simulation of 52 earthquake signals is performed to account for the uncertainty resulting from absence of precise knowledge of the actual ground motion. Structural performance may significantly change with respect to the type of earthquake. Additional seismological knowledge may reduce the earthquake uncertainty. However, such seismological considerations fall outside the scope of this paper.

\subsection{Results of structural identification}

In the following, the results of EDMF for the structural identification of the laboratory structure after earthquake excitation is presented. Fig. 7 shows EDMF performed 
(a)

Response spectra of earthquake signals (b)

Properties of earthquake signals
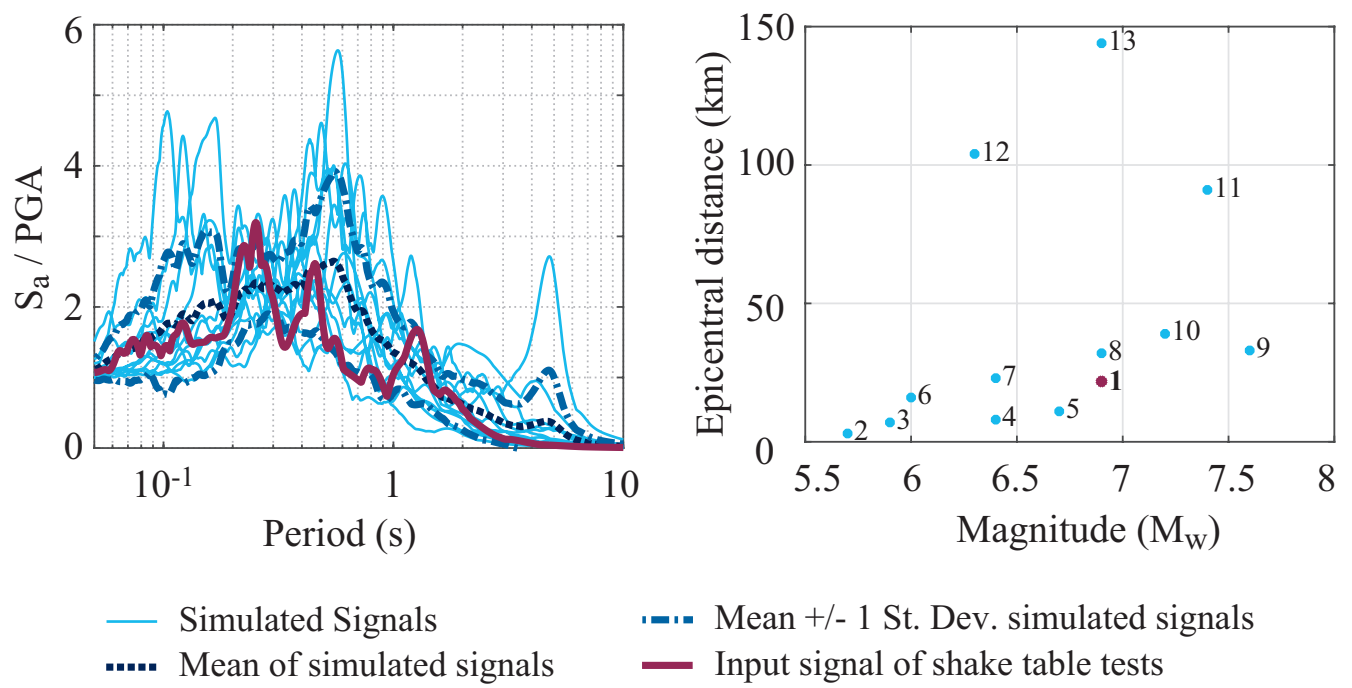

- Mean +/- 1 St. Dev. simulated signals
Input signal of shake table tests

Figure 6. Characteristics of the historic earthquake signals randomly chosen for time-history simulations. Acceleration response spectra (a) of the simulated signals are compared with the input signal for the shakingtable tests (damping is set at $5 \%$ ). St.Dev. is the standard deviation and $S_{a}$ is the spectral acceleration. Random selection of earthquakes is set to cover a large range of potential magnitudes and epicentral distances for the historic earthquakes (b).

on the post-earthquake fundamental frequency for the identification scenario $S_{4}$ (as defined in Table 2). Combining uncertainty sources results in a biased total uncertainty distribution. EDMF enables the engineer to define biased error distributions in a systematic and transparent manner, as described in the previous section.

In addition to the fundamental frequencies, DGs derived from visual inspection are used to falsify model instances. Fig. 8 provides an example of EDMF based on the observed DG (data interpretation event B in Fig. 2). Visual inspection performed after the shaking-table test revealed a $D G 2$. Translated to engineering quantities, a $D G 2$ means a maximum displacement included in the interval $\left[S_{d, 1}=0.7 \cdot D_{y} ; S_{d, 2}=1.5 \cdot D_{y}\right]$. The estimated uncertainties related to the displacement prediction and damage grade definitions (Table 4) define falsification thresholds for Eq. 7. Fig. 8 illustrates the identification scenario $S_{3}$, thus additional model instances are falsified based on the second available data source, the post-earthquake fundamental frequency, and may fall in between the thresholds for visual inspection. Fig. 7 and Fig. 8 are based on a target identification probability, $\phi_{d}=0.95$ (see Eq. 3).

The reduction that can be achieved in the number of model instances, and thus the reduction in parametric uncertainty, is most significant for scenario $S_{5}$, involving the most measured information, see Fig. 9. However, the improvement from two measured quantities to three measured quantities remains low. The Šidák correction (see Eq. 3) widens the threshold bounds when additional measurements are added. Also, redundancy in measured information can result in low increases in parametricuncertainty reduction. Therefore more information does not always result in more falsified model instances. As can be seen in Fig. 9, the observed DG is inefficient compared to frequency measurements in terms of model instance falsification based on a single measurement source. 


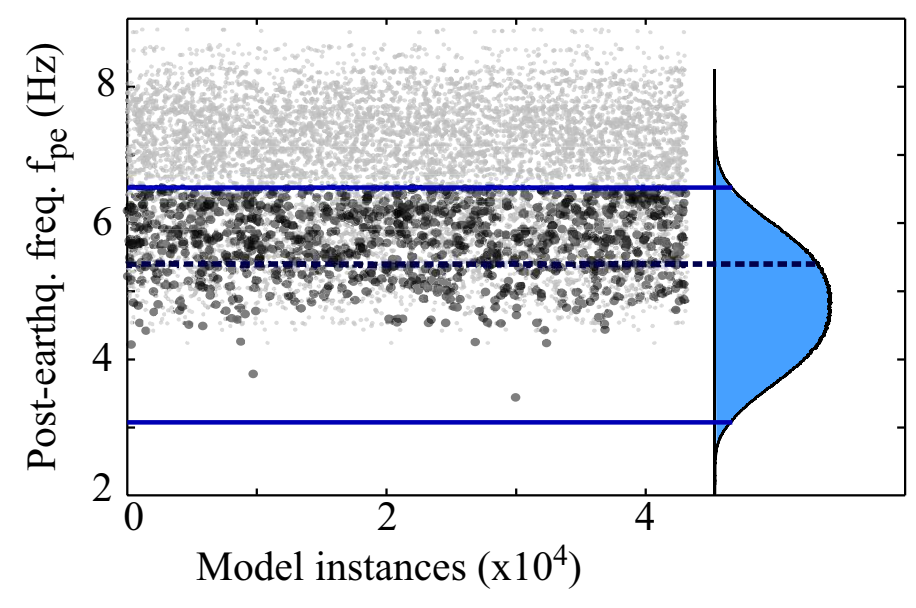

- Combined uncertainty

- Falsification threshold

--- Measured frequency

Falsified model instances

- Candidate model instances

Figure 7. EDMF plot based on post-earthquake natural frequency (Scenario $S_{4}$ ). Model instances that result in post-earthquake frequencies falling outside the thresholds are discarded.
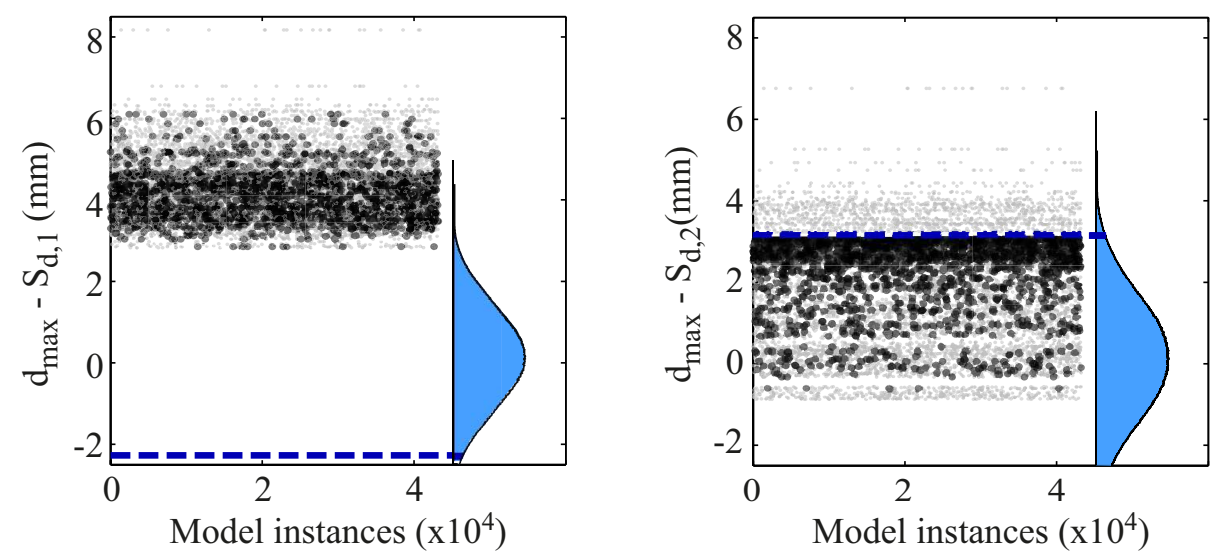

- Combined uncertainty - - Falsification threshold • Candidate models • Falsified models

Figure 8. EDMF plot for visual inspection (scenario $S_{3}$ ). A DG of 2 is observed after the shaking-table test. Candidate models need to be compatible with the observed DG (see Fig. 3). 
The earthquake uncertainty, related to either the intensity or the signal, is not significantly reduced through EDMF-based data interpretation, as only very few earthquake instances can be falsified (identical values of percentage of falsified model instances in Fig. 9 mean no signal instance is falsified). In addition, the reduction in parametric uncertainty that is not related to earthquake signals is significantly reduced when little information regarding the main shock is available. As shown in Fig. 10 for Scenario $S_{5}$, earthquakes with weaker PGA (divided by half) than the real earthquake are falsified for most earthquake signals (see Fig. 10). On the other hand, for earthquakes with higher PGA, parameter combinations exist that are compatible with all measured sources. No significant changes in the falsification performance can be observed with respect to magnitude or epicentral distance (see Fig. 6).

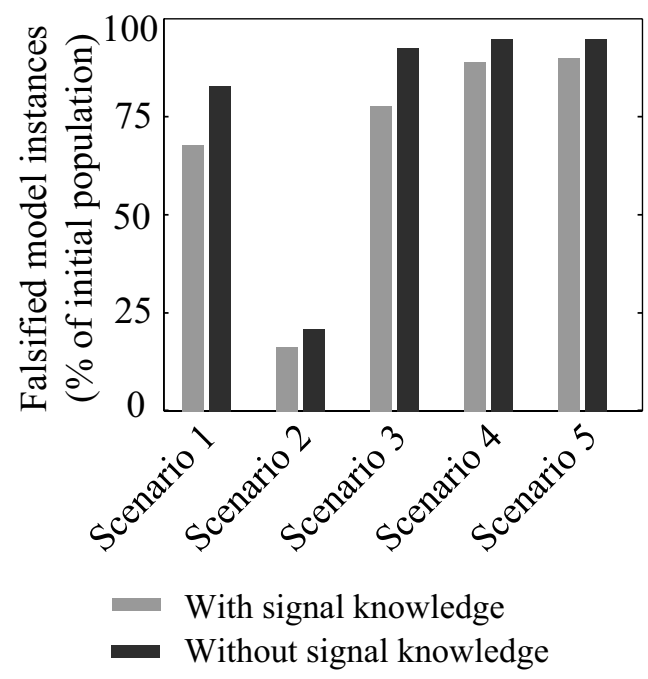

Figure 9. Percentage of model instances that is falsified based on the five identification scenarios that are defined in Table 1. Increase in identifiability between two measurements (Scenarios $\left(S_{3}\right.$ and $\left.S_{4}\right)$ and three measurements (Scenario $S_{5}$ ) is less than $10 \%$.

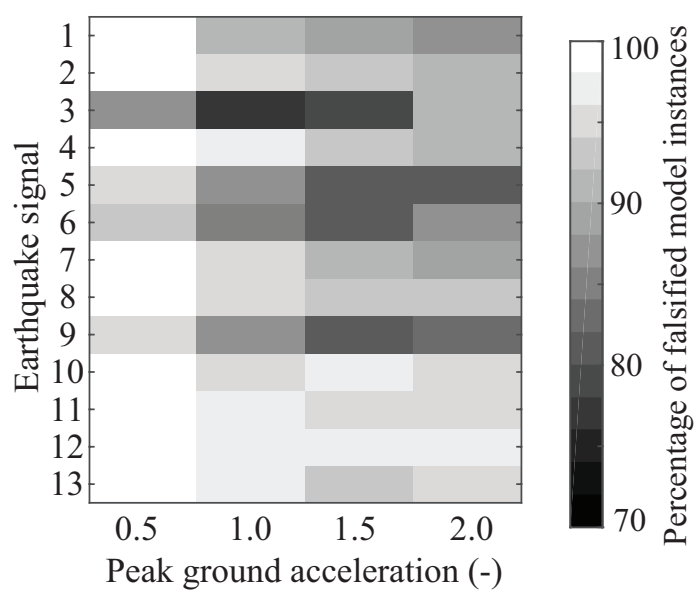

Figure 10. Percentage of initial model population that is falsified for the 52 earthquake signals that are simulated. Results are shown for Scenario $S_{5}$. Earthquakes with lower PGA are in general falsified (100\% of the model instances are discarded). However, for PGA values exceeding the value of the real earthquake, parameter combinations exist that are compatible with measurements. 


\subsection{Results of vulnerability prediction}

The candidate models are used to predict the maximum displacement that the structure undergoes during the subsequent shake-table tests. Fig. 11 summarizes the displacement predictions of the shake test that follows structural identification for the five identification scenarios. An average drift of $0.13 \%$ has been observed during the subsequent shaking test, characterized by a PGA of $3.6 \mathrm{~m} / \mathrm{s} 2$. The average drift is defined as the displacement of the top of the building (roof) divided by the total height of the structure. The prediction intervals in Fig. 11 correspond to a target prediction reliability $\left(\phi_{p}\right)$ of 0.99 . It is recalled that the total reliability for prediction has a lower bound of $\phi_{d} \cdot \phi_{p}$. A target identification reliability, $\phi_{d}$, of 0.95 is used in this case, thus an overall probability of 0.94 is obtained.

The parameter-based prediction uncertainty can be significantly reduced using structural identification. Indeed, for the chosen target reliability, the predicted displacement interval is reduced by 59 to 78 percent for the three shaking instances following structural identification when all measurement sources are used for falsification. The most significant reduction in the displacement prediction range is obtained for scenario $S_{2}$, visual inspection only, with reductions of up to $92 \%$. However, as can be seen in Fig. 11 , the prediction range for this scenario does not include the measured average drift that is used as a true reference value. Thus, relying on no measurement source except the observed DG may lead to potentially non-conservative predictions.

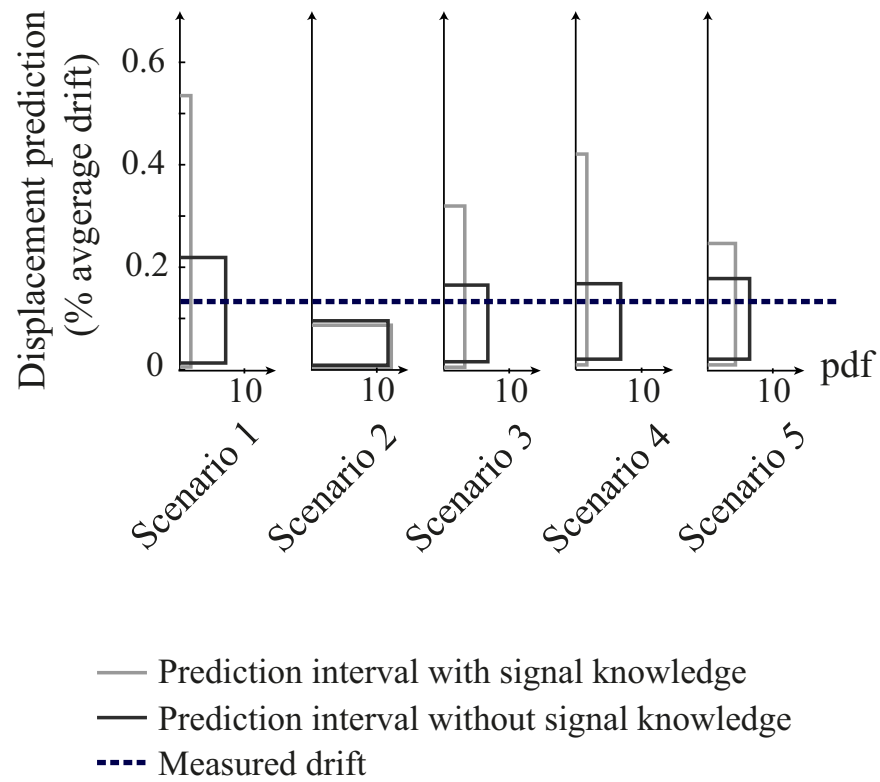

Figure 11. Displacement prediction intervals for the shake-table test following structural identification. Prediction intervals correspond to a target reliability of 0.94 .

Although the reduction in model instances resulting from visual inspection is the least efficient of all identification scenarios, the related prediction interval shows highest precision (narrow prediction interval). For the initial model population, most model instances predict low values of displacement and EDMF based on visual inspection retains most of these model instances as candidate models. Therefore, the observation that visual inspection alone outperforms any considerations of fundamental frequencies in terms of displacement prediction precision may be linked to the initial model population of this specific case study. 
Identification scenarios involving fundamental frequencies of the structure provide accurate predictions, as the measured drift is included in the predicted interval. In addition, the comparison between identification scenarios $S_{3}$ and $S_{5}$ in Fig. 11 shows that including the initial fundamental frequency does not provide a significant increase in the prediction precision. A similar tendency is observed between scenario $S_{1}$, based on the post-earthquake frequency, and scenario $S_{4}$ based on both fundamental frequencies, before and after the earthquake. Knowledge of the earthquake signal and intensity further improves the displacement-prediction precision and still provides robust predictions (see Fig. 11). This observation is valid for all the scenarios involving fundamental frequency measurements. As a conclusion, within the proposed framework, knowledge of ground-motion characteristics (through a dense seismic sensor network) is more important than knowledge of initial modal properties. In addition, as can be derived from scenario $S_{4}$, the methodology also applies to buildings that are equipped with devices with permanent monitoring, which measure initial and post-earthquake frequencies in addition to giving information on the main shock.

Estimating the model error involves engineering judgement and may therefore be seen as prone to subjectivity. However, the displacement prediction intervals for the studied structure show that EDMF provides robustness with respect to model-error estimations (Fig. 12). In Fig. 12, all model errors are varied linearly with respect to the estimated values presented in Table 4 . For a model error that is estimated at 25 percent of the values derived from engineering heuristics, all model instances are falsified. The falsification of the entire model population indicates either a wrong model class or a miss-evaluation of the modelling-error uncertainty distribution. Such a falsification of entire model populations enhances the robustness of data-interpretation methodologies with respect to inaccurate uncertainty estimations and cannot be achieved for structural identification methodologies relying on independent zero-mean normal uncertainty distributions (Pasquier \& Smith, 2015a; Reuland et al., 2017). Unless modelling error is taken as half of the estimated bounds, the measured average drift lies within the predicted interval, which is established for a target reliability of 0.94 .

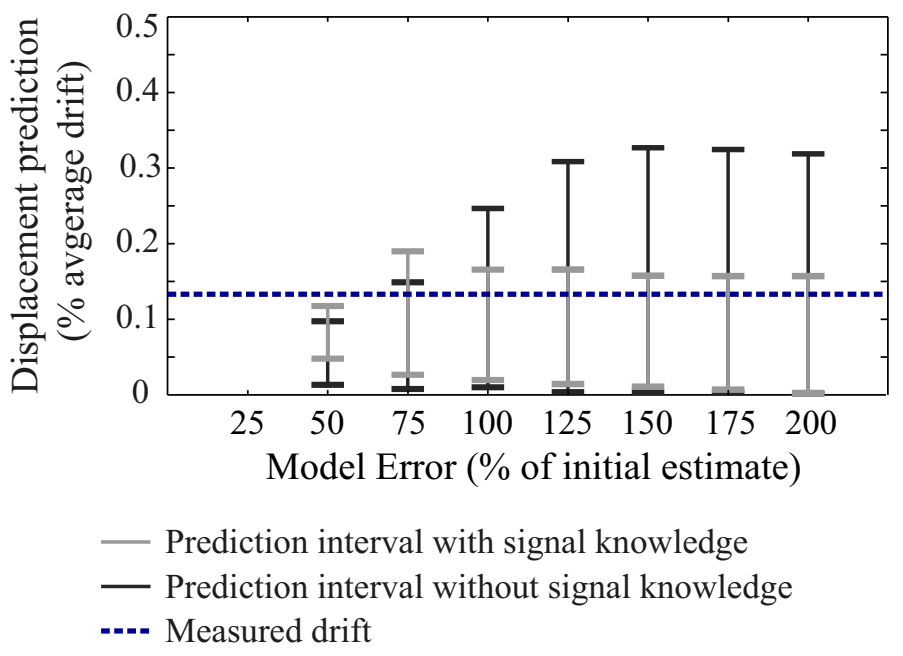

Figure 12. Influence of the estimated model error on displacement prediction intervals for the shaking-table test following structural identification. Prediction intervals correspond to a target reliability of 0.94 .

The inflection in the tendency of widening prediction ranges that can be observed around 75 percent for prediction intervals with signal knowledge and around 150 percent 
in absence of signal knowledge (see Fig. 12) results from the particular initial model population that predicts low displacement values with higher probability. Therefore, when the falsification thresholds are widened due to higher model errors, the prediction distribution shows higher probabilities for lower displacement values. This behaviour does not occur when prediction ranges are based on a target prediction reliability, $\phi_{p}$, taken equal to 1.

The displacement-demand estimation is an important parameter for vulnerability predictions of structures and therefore, the reduction in the prediction range that can be obtained through structural identification is promising. Data interpretation involving nonlinear time-history analyses based on a hysteretic Takeda SDOF model is shown to result in more precise, yet robust, structural-behaviour prognosis.

The final objective of structural identification in a post-earthquake context is to estimate the vulnerability of the building with regard to future earthquakes or seismic aftershocks. To meet this objective, identified candidate models are used to predict the probability of the building to reach a given DG for various earthquake amplitudes (see Fig 4). Since shake table tests are analysed in the case study, the DG for the three subsequent sequences is predicted instead of complete vulnerability curves.

The predicted DGs for the three subsequent shake-table tests at respectively 3.6, 6.3 and $14.8 \mathrm{~m} / \mathrm{s}^{2}$ are presented in Fig. 13. Similarly to the observations that are made regarding displacement predictions, candidate models that are identified using observed DGs lead to less conservative predictions than candidate models obtained from fundamental frequencies and may therefore result in non-conservative decision making. In addition, predictions based on the initial model population do not provide conservative estimates and thereby underline the importance of structural identification for post-earthquake assessment.

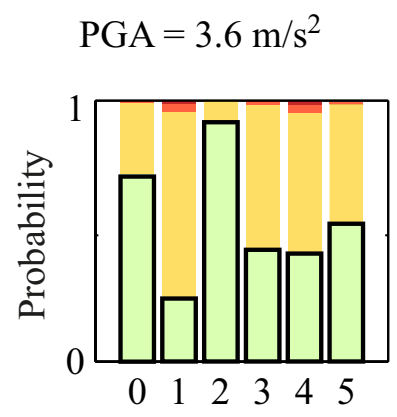

Scenario

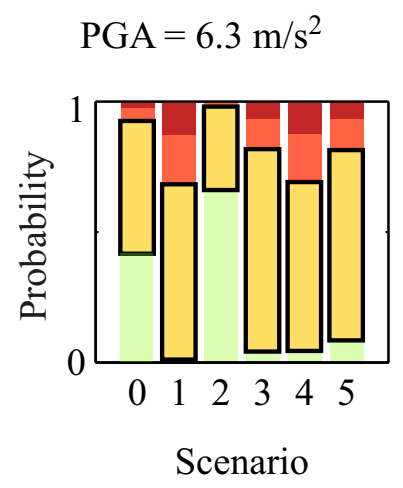

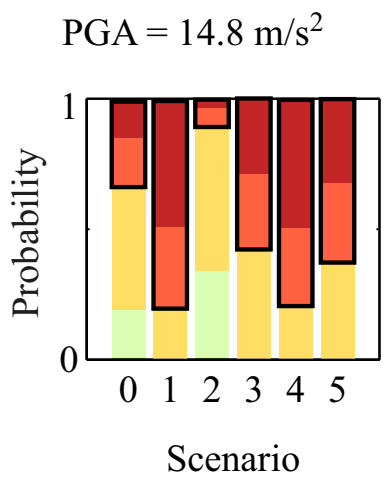

\footnotetext{
Damage Grade (DG) $2 \square$ Damage Grade (DG) 3

- Damage Grade (DG) $4 \quad$ Damage Grade (DG) 5
}

Figure 13. DG predictions for the three shake tests following identification. The DGs observed on the test specimen are highlighted with a dark border.

The DG prediction made with the candidate model set resulting from EDMF involving all three data sources predicts the correct DG with a probability of more than $50 \%$ for all three shaking events. The poorest result is obtained for the lowest excitation, where the candidate models predict a higher $D G$. As this prediction is conservative, this lack of precision is deemed acceptable.

As for displacement predictions, the availability of the undamaged fundamental 
frequency does not influence the predicted vulnerability. The predictions of scenarios $S_{1}$ and $S_{4}$ respectively $S_{3}$ and $S_{5}$ do not show any significant deviation. However, this observation may only apply to reinforced structures that show sufficient post-yield capacity and therefore loose stiffness gradually. The gradual decrease of the natural frequency observed during the shaking tests with increasing levels of actuation also indicates smooth stiffness reduction. Enhanced knowledge of the earthquake signal and amplitude only slightly improves the predictions, therefore the DG predictions with signal knowledge are not represented.

\section{Discussion and limitations}

The structure presented in the case study is a laboratory specimen. Therefore, several sources of uncertainties that would complicate the identification of full-scale structures in service are not present in this case study. Soil-structure interaction and uncertain stiffness contributions from secondary elements are, for example, not present in a laboratory environment. In addition, in real post-earthquake assessment campaigns, other factors (for example, debris and leakage) increase the measurement error. However, it is assumed that measurement errors will remain small in comparison with the modelling errors. This is also true for the effect of changing temperatures between two measurements, as long as boundary conditions are not changed by factors such as frozen soil. In addition, the structure was tested on a shake table and therefore, the excitation was well controlled and unidirectional, which limits torsional contributions to damage. The authors recognise that relying on a shake-table specimen (at half scale) provides an upper-bound estimate to the performance of the proposed methodology. However, as a first application, a test structure that has no additional uncertainty coming from non-structural walls is preferred, in order to provide a comprehensive comparison between model predictions and observed behaviour.

The conclusions drawn from the present work apply to one type of model (SDOF) and one type of building (mixed reinforced-concrete and unreinforced-masonry building). A simplified model (such as the SDOF model) presents multiple advantages and also, simulation time for large model populations remains acceptable. As the structural response is governed by a single hysteretic law, nonlinearity affects the entire structural response, while in more complex models, such as equivalent-frame models or multipledegree-of-freedom models, nonlinearity is often lumped into specific nodes. Therefore, damage (defined as a reduction in stiffness) has a global effect on structural responses (displacements and fundamental frequency) in such a SDOF model. The vertical distribution of cracks in mixed buildings makes the use of SDOF models an acceptable choice if uncertainties are taken into account. In addition, equivalent-frame models are known to have limited reliability in the elastic range and thus, for modal properties. To date, use of more complex models than SDOF has not been justified by superior results. Also, the goal of the proposed methodology is to identify residual seismic capacity, which needs a less refined model as compared to damage-detection applications. Nevertheless, extension to other models and influences of the choice of higher model complexity on simulation is the subject of ongoing work. 


\section{Conclusions}

A structural-identification strategy for earthquake-damaged buildings based on dynamic measurements and visual inspection is proposed. Parameter values of a simple physicsbased model are identified in order to enhance the estimations on how the building will behave during future seismic actions. Conclusions are as follows:

- Implementing EDMF reduces the uncertainty related to the state of damaged buildings by providing extrapolation predictions that are robust with respect to erroneous modelling-uncertainty evaluations and unknown uncertainty correlations. Therefore model-based data interpretation offers more precise estimations of the vulnerability of damaged buildings regarding future seismic actions compared with predictions that do not involve measurement-data interpretation.

- The methodology offers the possibility to simultaneously include information from subjective and objective data sources for the structural-identification task. The combination of visual inspection and ambient-vibration measurements leads to a robust identification of earthquake-damaged buildings.

- Visual inspection is a valuable tool to assess damaged structures. However, if it is the only source of information, erroneous conclusions may be drawn concerning the residual capacity of the structure because of high uncertainties related to the translation of visual damage grades to physical quantities, such as maximum displacements.

- EDMF allows the engineer to use simplified models to represent the structure, provided that the uncertainties resulting from omissions and idealizations are explicitly taken into account and combined in a transparent manner when falsification thresholds are calculated.

The results obtained for this case study show that the initial fundamental frequency, derived from measurements performed prior to an earthquake, did not contribute significantly to the uncertainty reduction. Future work involves investigating whether this promising finding is generalisable to similar building types and structural models.

\section{Acknowledgements}

The authors acknowledge EESD (Earthquake Engineering and Structural Dynamics Laboratory), EPFL for providing the test results, and K. Beyer for her remarks that helped to improve the quality of the work. This work was funded by the Swiss National Science Foundation under Contract No. 200020-169026.

\section{References}

Ambraseys, N., Smit, P., Douglas, J., Margaris, B., Sigbjrnsson, R., Olafsson, S., .. Costa, G. (2004). Internet site for European strong-motion data. Bollettino di Geofisica Teorica ed Applicata, 45(3), 113-129.

Astorga, A., Guéguen, P., \& Kashima, T. (2018). Nonlinear elasticity observed in buildings during a long sequence of earthquakes. Bulletin of the Seismological Society of America, 108(3A), 1185-1198.

Baggio, C., Bernardini, A., Colozza, R., Corazza, L., Della Bella, M., Di Pasquale, G., ... others (2007). Field manual for post-earthquake damage and safety assessment and short 
term countermeasures (aedes). European CommissionJoint Research CentreInstitute for the Protection and Security of the Citizen, EUR, 22868.

Balafas, K., \& Kiremidjian, A. S. (2015). Development and validation of a novel earthquake damage estimation scheme based on the continuous wavelet transform of input and output acceleration measurements. Earthquake Engineering \& Structural Dynamics, 44(4), 501-522.

Beck, J. L., \& Yuen, K.-V. (2004). Model selection using response measurements: Bayesian probabilistic approach. Journal of Engineering Mechanics, 130(2), 192-203.

Behmanesh, I., \& Moaveni, B. (2015, March). Probabilistic identification of simulated damage on the Dowling Hall footbridge through Bayesian finite element model updating. Structural Control and Health Monitoring, 22(3), 463-483.

Bensi, M., Kiureghian, A. D., \& Straub, D. (2014). Framework for post-earthquake risk assessment and decision making for infrastructure systems. ASCE-ASME Journal of Risk and Uncertainty in Engineering Systems, Part A: Civil Engineering, 1(1), 04014003.

Beyer, K., Tondelli, M., Petry, S., \& Peloso, S. (2015). Dynamic testing of a four-storey building with reinforced concrete and unreinforced masonry walls: prediction, test results and data set. Bulletin of Earthquake Engineering, 13(10), 3015-3064.

Calvi, G. M., Pinho, R., \& Crowley, H. (2006). State-of-the-knowledge on the period elongation of RC buildings during strong ground shaking. In PROC (CD) First European Conference on Earthquake Engineering and seismology, Geneva, Switzerland (pp. 3-8).

Chellini, G., De Roeck, G., Nardini, L., \& Salvatore, W. (2010). Damage analysis of a steelconcrete composite frame by finite element model updating. Journal of Constructional Steel Research, 66(3), 398-411.

Clinton, J. F., Bradford, S. C., Heaton, T. H., \& Favela, J. (2006). The observed wander of the natural frequencies in a structure. Bulletin of the Seismological Society of America, 96(1), $237-257$.

D'Ayala, D. F., \& Paganoni, S. (2010). Assessment and analysis of damage in L'Aquila historic city centre after 6th April 2009. Bulletin of Earthquake Engineering, 9(1), 81-104.

Dunand, F., Ait Meziane, Y., Guéguen, P., Chatelain, J.-L., Guillier, B., Ben Salem, R., .. . others (2004). Utilisation du bruit de fond pour l'analyse des dommages des bâtiments de boumerdes suite au séisme du 21 mai 2003. Mémoires du Service Géologique de l'Algérie, 12, 177-191.

Elwood, K., Marder, K., Pampanin, S., Ramirez, A. C., Kral, M., Smith, P., .. Stannard, M. (2016). Draft framework for assessing residual capacity of earthquake-damaged concrete buildings. In Proceedings of the annual new zealand society for earthquake engineering (NZSEE) Conference, April 1-3, 2016, Christchurch, New Zealand.

Enright, M. P., \& Frangopol, D. M. (1999). Condition prediction of deteriorating concrete bridges using Bayesian updating. Journal of Structural Engineering, 125(10), 1118-1125.

Esteva, L., Díaz-López, O. J., Vásquez, A., \& León, J. A. (2016). Structural damage accumulation and control for life cycle optimum seismic performance of buildings. Structure and Infrastructure Engineering, 12(7), 848-860.

Galloway, B., Hare, J., Brunsdon, D., Wood, P., Lizundia, B., \& Stannard, M. (2014). Lessons from the Post-Earthquake Evaluation of Damaged Buildings in Christchurch. Earthquake Spectra, 30(1), 451-474.

Gentile, A., \& Messina, A. (2003). On the continuous wavelet transforms applied to discrete vibrational data for detecting open cracks in damaged beams. International Journal of Solids and Structures, 40(2), 295-315.

Ghosh, J., Padgett, J. E., \& Sánchez-Silva, M. (2015). Seismic damage accumulation in highway bridges in earthquake-prone regions. Earthquake Spectra, 31(1), 115-135.

Goretti, A., \& Di Pasquale, G. (2002). An overview of post-earthquake damage assessment in Italy. In Eeri invitational workshop. An action plan to develop earthquake damage and loss data protocols, California.

Goretti, A., Hutt, C. M., \& Hedelund, L. (2017). Post-earthquake safety evaluation of buildings in portoviejo, manabí province, following the mw7. 8 ecuador earthquake of april 16, 2016 International journal of disaster risk reduction, 24, 271-283. 
Goulet, J.-A., Michel, C., \& Kiureghian, A. D. (2015). Data-driven post-earthquake rapid structural safety assessment. Earthquake Engineering \& Structural Dynamics, 44(4), 549562.

Goulet, J.-A., Michel, C., \& Smith, I. F. C. (2013). Hybrid probabilities and error-domain structural identification using ambient vibration monitoring. Mechanical Systems and Signal Processing, 37(1-2), 199-212.

Goulet, J.-A., \& Smith, I. F. C. (2013). Structural identification with systematic errors and unknown uncertainty dependencies. Computers $\&$ Structures, 128, 251-258.

Grünthal, G., Musson, R. M. V., Schwarz, J., \& Stucchi, M. (2001). European Macroseismic Scale 1998, EMS-98. Cahiers du Centre Européen de Géodynamique et de Séismologie, Volume 19.

Huang, C. S., Hung, S. L., Wen, C. M., \& Tu, T. T. (2003). A neural network approach for structural identification and diagnosis of a building from seismic response data. Earthquake Engineering 8 Structural Dynamics, 32(2), 187-206.

Iervolino, I., Giorgio, M., \& Chioccarelli, E. (2014). Closed-form aftershock reliability of damage-cumulating elastic-perfectly-plastic systems. Earthquake Engineering \&5 Structural Dynamics, 43(4), 613-625.

Jalayer, F., Asprone, D., Prota, A., \& Manfredi, G. (2011). A decision support system for post-earthquake reliability assessment of structures subjected to aftershocks: an application to laquila earthquake, 2009. Bulletin of Earthquake Engineering, 9(4), 997-1014.

Jalayer, F., \& Ebrahimian, H. (2017). Seismic risk assessment considering cumulative damage due to aftershocks. Earthquake Engineering \& Structural Dynamics, 46(3), 369-389.

Jeon, J.-S., DesRoches, R., Lowes, L. N., \& Brilakis, I. (2015). Framework of aftershock fragility assessment-case studies: older california reinforced concrete building frames. Earthquake Engineering \& Structural Dynamics, 44(15), 2617-2636.

Katsanos, E. I., Sextos, A. G., \& Elnashai, A. S. (2014). Prediction of inelastic response periods of buildings based on intensity measures and analytical model parameters. Engineering Structures, 71, 161-177.

Lagomarsino, S., \& Giovinazzi, S. (2006). Macroseismic and mechanical models for the vulnerability and damage assessment of current buildings. Bulletin of Earthquake Engineering, 4(4), 415-443.

Lestuzzi, P., Belmouden, Y., \& Trueb, M. (2007). Non-linear seismic behavior of structures with limited hysteretic energy dissipation capacity. Bulletin of Earthquake Engineering, 5(4), 549-569.

Maity, D., \& Saha, A. (2004). Damage assessment in structure from changes in static parameter using neural networks. Sadhana, 29(3), 315-327.

Marquis, F., Kim, J. J., Elwood, K. J., \& Chang, S. E. (2017). Understanding post-earthquake decisions on multi-storey concrete buildings in Christchurch, New Zealand. Bulletin of earthquake engineering, 15(2), 731-758.

Marshall, J. D., Jaiswal, K., Gould, N., Turner, F., Lizundia, B., \& Barnes, J. C. (2013). Post-Earthquake Building Safety Inspection: Lessons from the Canterbury, New Zealand, Earthquakes. Earthquake Spectra, $29(3), 1091-1107$.

Michel, C., Zapico, B., Lestuzzi, P., Molina, F. J., \& Weber, F. (2011). Quantification of fundamental frequency drop for unreinforced masonry buildings from dynamic tests. Earthquake Engineering 85 Structural Dynamics, 40(11), 1283-1296.

Mitrani-Resier, J., Wu, S., \& Beck, J. L. (2016). Virtual inspector and its application to immediate pre-event and post-event earthquake loss and safety assessment of buildings. Natural Hazards, 81 (3), 1861-1878.

Moaveni, B., Conte, J. P., \& Hemez, F. M. (2009). Uncertainty and sensitivity analysis of damage identification results obtained using finite element model updating. Computer-Aided Civil and Infrastructure Engineering, 24(5), 320-334.

Moaveni, B., He, X., Conte, J. P., \& Restrepo, J. I. (2010). Damage identification study of a seven-story full-scale building slice tested on the UCSD-NEES shake table. Structural Safety, 32(5), 347-356. 
Mucciarelli, M., Masi, A., Gallipoli, M. R., Harabaglia, P., Vona, M., Ponzo, F., \& Dolce, M. (2004). Analysis of RC building dynamic response and soil-building resonance based on data recorded during a damaging earthquake (Molise, Italy, 2002). Bulletin of the Seismological Society of America, 94(5), 1943-1953.

Özer, E., \& Soyöz, S. (2013, September). Vibration-Based Damage Detection and Seismic Performance Assessment of Bridges. Earthquake Spectra, 31(1), 137-157.

Pai, S. G. S., Nussbaumer, A., \& Smith, I. F. C. (2018). Comparing structural identification methodologies for fatigue life prediction of a highway bridge. Frontiers in Built Environment, 3, 73 .

Paparo, A., \& Beyer, K. (2013). Seismic behaviour of mixed rc-urm wall structures: comparison between numerical results and experimental evidence. In Proceedings of the vienna congress on recent advances in earthquake engineering and structural dynamics.

Paparo, A., \& Beyer, K. (2014). Quasi-static cyclic tests of two mixed reinforced concreteunreinforced masonry wall structures. Engineering Structures, 71, 201-211.

Pasquier, R., \& Smith, I. F. (2015b). Sources and forms of modelling uncertainties for structural identification. In 7th International Conference on Structural Health Monitoring of Intelligent Infrastructure (SHMII).

Pasquier, R., \& Smith, I. F. C. (2015a). Robust system identification and model predictions in the presence of systematic uncertainty. Advanced Engineering Informatics, 29(4), 1096-1109.

Pasquier, R., \& Smith, I. F. C. (2016). Iterative structural identification framework for evaluation of existing structures. Engineering Structures, 106, 179-194.

Polese, M., Di Ludovico, M., Marcolini, M., Prota, A., \& Manfredi, G. (2015). Assessing reparability: simple tools for estimation of costs and performance loss of earthquake damaged reinforced concrete buildings. Earthquake Engineering \& Structural Dynamics, 44(10), 1539-1557.

Popper, K. R. (1959). The logic of scientific discovery. London: Hutchinson.

Raghunandan, M., Liel, A. B., \& Luco, N. (2015). Aftershock collapse vulnerability assessment of reinforced concrete frame structures. Earthquake Engineering 8 Structural Dynamics, $44(3), 419-439$.

Raphael, B., \& Smith, I. F. C. (1998). Finding the right model for bridge diagnosis. In Artificial intelligence in structural engineering (pp. 308-319). Springer.

Reuland, Y., Lestuzzi, P., \& Smith, I. F. C. (2017). Data-interpretation methodologies for non-linear earthquake response predictions of damaged structures. Frontiers in Built Environment, 3, 43.

Robert-Nicoud, Y., Raphael, B., \& Smith, I. F. C. (2005). System identification through model composition and stochastic search. Journal of computing in civil engineering, 19(3), 239-247.

Shiradhonkar, S., \& Shrikhande, M. (2011). Seismic damage detection in a building frame via finite element model updating. Computers \& Structures, 89(23-24), 2425-2438.

Smith, I. F. C. (2016). Studies of Sensor-Data Interpretation for Asset Management of the Built Environment. Frontiers in Built Environment, 2, 8.

Takeda, T., Sozen, M. A., \& Nielsen, N. N. (1970). Reinforced concrete response to simulated earthquakes. Journal of the Structural Division, 96(12), 2557-2573.

Trevlopoulos, K., \& Guéguen, P. (2016). Period elongation-based framework for operative assessment of the variation of seismic vulnerability of reinforced concrete buildings during aftershock sequences. Soil Dynamics and Earthquake Engineering, 84, 224-237.

Vidal, F., Navarro, M., Aranda, C., \& Enomoto, T. (2013). Changes in dynamic characteristics of Lorca RC buildings from pre-and post-earthquake ambient vibration data. Bulletin of Earthquake Engineering, 1-16.

Vona, M., Cascini, G., Mastroberti, M., Murgante, B., \& Nolè, G. (2017). Characterization of urm buildings and evaluation of damages in a historical center for the seismic risk mitigation and emergency management. International Journal of Disaster Risk Reduction, 24, 251-263.

Šidák, Z. (1967). Rectangular confidence regions for the means of multivariate normal distributions. Journal of the American Statistical Association, 62(318), 626-633. 
Yazgan, U., \& Dazio, A. (2012). Post-earthquake damage assessment using residual displacements. Earthquake Engineering \&3 Structural Dynamics, 41(8), 1257-1276.

Yeo, G. L., \& Cornell, C. A. (2009). Post-quake decision analysis using dynamic programming. Earthquake Engineering 83 Structural Dynamics, 38(1), 79-93.

Zapico, J. L., \& Gonzalez, M. P. (2006). Numerical simulation of a method for seismic damage identification in buildings. Engineering Structures, 28(2), 255-263.

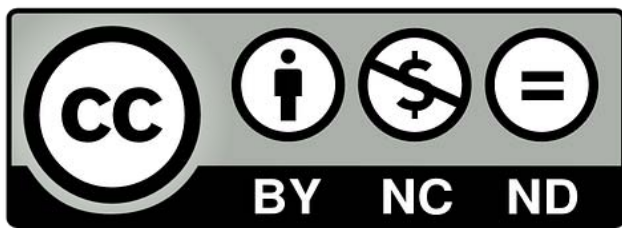

